\title{
Surface Hopping Propagator: An Alternative Approach to Diffusion-Influenced Reactions
}

\author{
Denis S. Grebenkov ${ }^{1, *}$ \\ 1 Laboratoire de Physique de la Matière Condensée (UMR 7643), \\ CNRS - Ecole Polytechnique, IP Paris, 91128 Palaiseau, France
}

(Dated: August 28, 2020)

\begin{abstract}
Dynamics of a particle diffusing in a confinement can be seen a sequence of bulk-diffusion-mediated hops on the confinement surface. Here, we investigate the surface hopping propagator that describes the position of the diffusing particle after a prescribed number of encounters with that surface. This quantity plays the central role in diffusion-influenced reactions and determines their most common characteristics such as the propagator, the first-passage time distribution, and the reaction rate. We derive explicit formulas for the surface hopping propagator and related quantities for several Euclidean domains: half-space, circular annuli, circular cylinders, and spherical shells. These results provide the theoretical ground for studying diffusion-mediated surface phenomena. The behavior of the surface hopping propagator is investigated for both "immortal" and "mortal" particles.
\end{abstract}

PACS numbers: 02.50.-r, 05.40.-a, 02.70.Rr, 05.10.Gg

Keywords: diffusion, escape problem, first passage time, mixed boundary condition

\section{INTRODUCTION}

In many natural phenomena, particles diffuse in a confinement towards its surface where they can react, permeate, relax their activity or be killed. Examples include heterogeneous catalysis, permeation across cell membranes, filtering in porous media, surface relaxation in nuclear magnetic resonance, and animal foraging [1-9]. These phenomena are conventionally described by diffusion equation (or more general Fokker-Planck equation) with appropriate boundary conditions [10, 11]. In particular, most common properties of diffusion-influenced reactions are derived from the propagator $G_{q}\left(\boldsymbol{x}, t \mid \boldsymbol{x}_{0}\right)$, i.e., the probability density of the event that a particle, started from a bulk point $\boldsymbol{x}_{0}$ at time 0 , has not reacted on the surface and located at a bulk point $\boldsymbol{x}$ at time $t$. For normal diffusion, the propagator satisfies the diffusion equation inside a confining domain $\Omega$

$$
\partial_{t} G_{q}\left(\boldsymbol{x}, t \mid \boldsymbol{x}_{0}\right)=D \Delta_{\boldsymbol{x}} G_{q}\left(\boldsymbol{x}, t \mid \boldsymbol{x}_{0}\right) \quad(\boldsymbol{x} \in \Omega),
$$

subject to the initial condition $G_{q}\left(\boldsymbol{x}, t=0 \mid \boldsymbol{x}_{0}\right)=\delta(\boldsymbol{x}-$ $\left.\boldsymbol{x}_{0}\right)$ and the Robin boundary condition on the boundary $\partial \Omega$ :

$$
-\partial_{n} G_{q}\left(\boldsymbol{x}, t \mid \boldsymbol{x}_{0}\right)=q G_{q}\left(\boldsymbol{x}, t \mid \boldsymbol{x}_{0}\right) \quad(\boldsymbol{x} \in \partial \Omega),
$$

where $\Delta_{\boldsymbol{x}}$ is the Laplace operator acting on $\boldsymbol{x}, \delta(\boldsymbol{x})$ is the Dirac distribution, and $\partial_{\boldsymbol{n}}$ is the normal derivative on the boundary $\partial \Omega$ oriented outwards the domain $\Omega$. The parameter $q=\kappa / D$ is the ratio between the surface reactivity (or permeability, or relaxivity, etc.) $\kappa$ and bulk diffusivity $D$. In chemical physics, the Robin boundary condition was put forward by Collins and Kimball [12] and later explored by many researchers [13-32] (see an

*Electronic address: denis.grebenkov@polytechnique.edu overview in [33]). The major disadvantage of the conventional description is that the surface reactivity $\kappa$ (or $q$ ) enters implicitly as a parameter of the Robin boundary condition (2).

In a recent work [34], we proposed an alternative description based on the concept of boundary local time. The boundary local time $\ell_{t}$ characterizes the fraction of time that a diffusing particle spends in a close vicinity of the reflecting boundary, as well as the number of encounters with that boundary [35], see Eqs. (4, 5) below. This is a fundamental concept in the theory of stochastic processes [36, 37], which remains largely unknown and almost unemployed in physics, chemistry and biology. To incorporate $\ell_{t}$, we introduced the full propagator $P\left(\boldsymbol{x}, \ell, t \mid \boldsymbol{x}_{0}\right)$, i.e., the joint probability density of finding a particle at point $\boldsymbol{x}$ at time $t$ with its boundary local time $\ell$, given that it started from $\boldsymbol{x}_{0}$ at time 0 . The crucial advantage of this alternative description is that $P\left(\boldsymbol{x}, \ell, t \mid \boldsymbol{x}_{0}\right)$ characterizes diffusion in confinement with reflecting (inert) boundary. In turn, the surface reactivity is introduced via a stopping condition on the boundary local time. In particular, we derived

$$
G_{q}\left(\boldsymbol{x}, t \mid \boldsymbol{x}_{0}\right)=\int_{0}^{\infty} d \ell e^{-q \ell} P\left(\boldsymbol{x}, \ell, t \mid \boldsymbol{x}_{0}\right),
$$

where the surface reactivity $q$ appears explicitly as a parameter of the Laplace transform with respect to the boundary local time $\ell$. In this way, the single full propagator $P\left(\boldsymbol{x}, \ell, t \mid \boldsymbol{x}_{0}\right)$ describes the whole family of partially reactive surfaces (characterized by $q$ ). Moreover, one can replace the exponential factor $e^{-q \ell}$ by a more general function to implement other surface reaction mechanisms far beyond the conventional partial reactivity described by the Robin boundary condition (2), see [34] for details. In this light, the full propagator $P\left(\boldsymbol{x}, \ell, t \mid \boldsymbol{x}_{0}\right)$ turns out to be the intrinsic key quantity that describes all sorts 
of diffusion-mediated surface phenomena in a given confinement.

A successful implementation of this new paradigm requires efficient methods for accessing the full propagator. In [34], the Laplace transform of the full propagator was expressed in terms of the so-called "surface hopping propagator" $\Sigma_{p}\left(\boldsymbol{s}, \ell \mid \boldsymbol{s}_{0}\right)$, i.e., the probability density of the event that a particle, started from a boundary point $s_{0}$, has survived against a "bulk killing" with the rate $p$ and located at a boundary point $s$ at the boundary local time $\ell$. The rate $p \geq 0$ accounts for eventual disappearance of the particles during its diffusion in the domain $\Omega$ due to a bulk reaction or spontaneous disintegration, relaxation, photobleaching or death. In this scheme, one can consider both "mortal" $(p>0)$ and "immortal" $(p=0)$ particles [38-40]. In other words, the surface hopping propagator describes bulk-diffusion-mediated displacements between two encounters with the boundary, separated by the boundary local time $\ell$. The concept of such a surface exploration by successive hops through the bulk was formulated by Bychuk and O'Shaugnessy [41, 42] and later confirmed by single-particle tracking experiments [43-45]. Former theoretical descriptions of surface hopping diffusion in terms of effective surface propagators were based on coupled bulk-surface diffusion equations with adsorption/desorption kinetics [46-50]. In turn, the surface hopping propagator $\Sigma_{p}\left(\boldsymbol{s}, \ell \mid \boldsymbol{s}_{0}\right)$ is a conceptually different quantity, which characterizes surface displacements not in terms of physical time $t$ (as earlier) but in terms of the boundary local time $\ell$ (the number of encounters). To our knowledge, the surface hopping propagator, introduced in [34] as an efficient way to access the full propagator, is a new object, and the present paper aims at uncovering its properties.

The paper is organized as follows. In Sec. II, we formulate the theoretical framework for diffusion-mediated surface phenomena, build an intuitive ground for the surface hopping propagator, and recall some general relations from [34]. Main results are reported in Sec. III, in which the surface hopping propagator is computed and investigated for several domains. Section IV summarizes and concludes the paper.

\section{SURFACE HOPPING PROPAGATOR}

How many reflections does a particle undertake up to a given time $t$ or during its lifetime? Where is the particle after $n$ reflections? For the common continuoustime Brownian motion, these natural questions have old but disappointing (trivial) answers. In fact, Brownian motion crossing a smooth surface is known to return infinitely many times to that surface within an infinitely short time period [51]. To get more satisfactory answers, one needs to reformulate these questions in a regularized way. For instance, one can substitute Brownian motion by a sequence of independent jumps (e.g., a random walk on a lattice). However, it is more con- venient to keep considering continuous stochastic process $\boldsymbol{X}_{t}$ but to introduce a thin surface layer of width $a$, $\partial \Omega_{a}=\{\boldsymbol{x} \in \Omega:|\boldsymbol{x}-\partial \Omega|<a\}$, and to count the number $\mathcal{N}_{t}^{a}$ of crossings of this layer by reflected Brownian motion up to time $t$. As $a \rightarrow 0$, the number of crossings diverges but $a \mathcal{N}_{t}^{a}$ converges to the random process, introduced by Lévy and called the boundary local time [35]:

$$
\ell_{t}=\lim _{a \rightarrow 0} a \mathcal{N}_{t}^{a} .
$$

While the continuous time $t$ represents the number of jumps of duration $\delta$ that the particle undertakes in the bulk, the boundary local time $\ell$ is the proxy of the number of encounters with the boundary (reflections of amplitude $a$ ). Equivalently, $\ell_{t}$ is proportional to the fraction of time that a particle spent in the surface layer of width $a$ up to time $t$ :

$$
\ell_{t}=\lim _{a \rightarrow 0} \frac{D}{a} \int_{0}^{t} d t^{\prime} \mathbb{I}_{\partial \Omega_{a}}\left(\boldsymbol{X}_{t^{\prime}}\right),
$$

where the integral is the residence time of reflected Brownian motion $\boldsymbol{X}_{t}$ in $\partial \Omega_{a}$, and $\mathbb{I}_{\partial \Omega_{a}}(\boldsymbol{x})$ is the indicator function of that layer: $\mathbb{I}_{\partial \Omega_{a}}(\boldsymbol{x})=1$ for $\boldsymbol{x} \in \partial \Omega_{a}$, and 0 otherwise. While $\ell_{t}$ is historically called "local time", it has units of length (see also [52, 53]). In some definitions, the diffusion coefficient $D$ is removed from Eq. (5), yielding the boundary local time in units of time per length, i.e., the time spent in the surface layer rescaled by its width. We also stress that the boundary local time should not be confused with a closely related notion of the point local time, i.e., a fraction of time spent in an infinitesimal vicinity of a fixed bulk point. The latter was thoroughly investigated, in particular, for Brownian motion and Bessel processes (see [54-56] and references therein).

The former two questions should thus be reformulated in terms of the boundary local time: How large is the boundary local time $\ell_{t}$ up to a given time $t$ or during the lifetime of a particle? Where is the particle after a boundary local time $\ell$ ? Answers to both these questions are given by the surface hopping propagator $\Sigma_{p}\left(s, \ell \mid s_{0}\right)$, as discussed below.

\section{A. Intuitive picture}

Before presenting main results for general domains, it is instructive to provide the motivation and intuition for the surface hopping propagator. Let us consider a particle diffusing in the upper half-plane, $\Omega=\mathbb{R} \times \mathbb{R}_{+}$, with reflecting horizontal axis $\partial \Omega=\{(x, 0): x \in \mathbb{R}\}$. If the particle started from a boundary point $\boldsymbol{s}_{0}=\left(x_{0}, 0\right)$, its "next" encounter with the boundary would occur exactly at $\boldsymbol{s}_{0}$, as discussed above. To overcome this problem, we introduce a thin surface layer of width $a$ (Fig. 1). Now, one can ask what is the position of the next encounter 
with the boundary after crossing the horizontal line $y=a$ (i.e., after exiting from the surface layer). This regularization eliminates too short Brownian trajectories that remain within the layer. As the first crossing of the line $y=a$ typically occurs near the starting point $s_{0}$, one can move the starting point from $\left(x_{0}, 0\right)$ to $\left(x_{0}, a\right)$ and then search for the probability density of the first arrival onto the horizontal axis. This is the harmonic measure density, which for the upper half-plane takes the form of the Cauchy density,

$$
p_{1}\left(x \mid\left(x_{0}, a\right)\right)=\frac{a}{\pi\left[\left(x-x_{0}\right)^{2}+a^{2}\right]},
$$

and can thus describe the first encounter position $x$ after leaving the boundary from $x_{0}$ and crossing the surface layer of width $a$. After this encounter, the particle continues diffusion, independently of its past, so that the second encounter position is determined by the convolution of two Cauchy densities:

$$
\begin{aligned}
p_{2}\left(x \mid\left(x_{0}, a\right)\right) & =\int_{\mathbb{R}} d x_{1} p_{1}\left(x \mid\left(x_{1}, a\right)\right) p_{1}\left(x_{1} \mid\left(x_{0}, a\right)\right) \\
& =\frac{2 a}{\pi\left[\left(x-x_{0}\right)^{2}+(2 a)^{2}\right]} .
\end{aligned}
$$

Similarly, the position of the $n$-th encounter is determined by

$$
p_{n}\left(x \mid\left(x_{0}, a\right)\right)=\frac{n a}{\pi\left[\left(x-x_{0}\right)^{2}+(n a)^{2}\right]} .
$$

In the limit $a \rightarrow 0$ with any fixed $n$, this density converges to the Dirac distribution, $p_{n}\left(x \mid\left(x_{0}, a\right)\right) \rightarrow \delta\left(x-x_{0}\right)$, illustrating the above statement that (reflected) Brownian motion returns infinitely many times to the first hitting point within an infinitely short period. As the right-hand side of Eq. (7) depends on $a$ via the product na, a nontrivial result can only be obtained in the limit $a \rightarrow 0$ when $n a$ is fixed. Setting $\ell=n a$, one obtains the surface hopping propagator for the upper half-plane:

$$
\Sigma_{0}\left(s, \ell \mid s_{0}\right)=\frac{\ell}{\pi\left[\left(x-x_{0}\right)^{2}+\ell^{2}\right]},
$$

with boundary points $s=(x, 0)$ and $\boldsymbol{s}_{0}=\left(x_{0}, 0\right)$. As eventual death of the particle during its bulk diffusion was ignored, we set $p=0$ in the subscript.

While the above construction can be performed in any confining domain, its practical realization involves numerous convolutions of the harmonic measure density which in general are difficult to compute (the above explicit computation was possible due to the explicit form of the Cauchy distribution and its specific "infinite divisibility" property, i.e., the invariance of its form upon convolutions). In the next subsection, we present a general approach to access the surface hopping propagator.

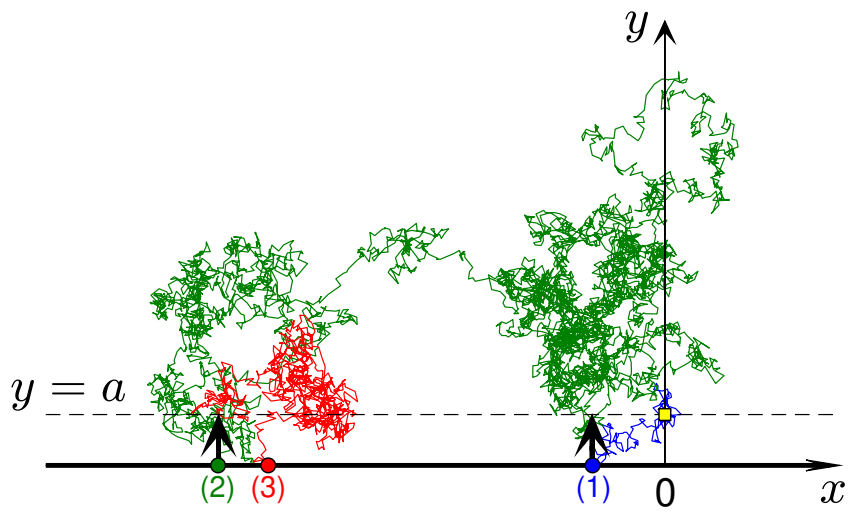

FIG. 1: Simulated trajectory of Brownian motion in the upper half-plane above the horizontal axis. A thin surface layer is delimited by dashed line at $y=a$. The trajectory, started from $(0, a)$ (yellow filled square) is split into three colored parts (blue, green, red). Each part is terminated when the particle hits the boundary (enumerated filled circles), while the next part starts the distance $a$ above the last hitting point ("jumps" indicated by black arrows). While some bulk explorations are short (blue and red parts), the other can be very long (green part).

\section{B. General approach}

We consider a particle diffusing in an Euclidean domain $\Omega \subset \mathbb{R}^{d}$ with a smooth boundary $\partial \Omega$. In [34], the surface hopping propagator $\Sigma_{p}\left(\boldsymbol{s}, \ell \mid \boldsymbol{s}_{0}\right)$ (with $p \geq 0$ ) was shown to be the kernel of the semi-group $\exp \left(-\mathcal{M}_{p} \ell\right)$ generated by the Dirichlet-to-Neumann operator $\mathcal{M}_{p}$. This is a pseudo-differential self-adjoint operator acting on functions on the boundary $\partial \Omega$ (see rigorous definitions and mathematical details in [57-61]). For a given function $f$ on $\partial \Omega$, this operator associates another function $g$ on $\partial \Omega, \mathcal{M}_{p}: f \rightarrow g=\left(\partial_{\boldsymbol{n}} w\right)_{\mid \partial \Omega}$, where $w$ satisfies the Dirichlet boundary value problem:

$$
(p-D \Delta) w=0 \quad \text { in } \Omega, \quad w_{\mid \partial \Omega}=f
$$

(if $\Omega$ is unbounded, one also needs to impose the regularity condition: $w \rightarrow 0$ as $|\boldsymbol{x}| \rightarrow \infty$, see below). For instance, if $f$ describes a concentration of particles maintained on the boundary $\partial \Omega$, then $\mathcal{M}_{p} f=\left(\partial_{\boldsymbol{n}} w\right)_{\mid \partial \Omega}$ is proportional to the diffusive flux of these particles into the bulk. Note that there is a family of Dirichlet-toNeumann operators parameterized by $p$ (or $p / D$ ). As the kernel of the semi-group $\exp \left(-\mathcal{M}_{p} \ell\right)$, the surface hopping propagator satisfies

$$
\partial_{\ell} \Sigma_{p}\left(\boldsymbol{s}, \ell \mid \boldsymbol{s}_{0}\right)=-\mathcal{M}_{p} \Sigma_{p}\left(\boldsymbol{s}, \ell \mid \boldsymbol{s}_{0}\right),
$$

subject to the initial condition $\Sigma_{p}\left(s, \ell=0 \mid s_{0}\right)=\delta(s-$ $\left.\boldsymbol{s}_{0}\right)$. This equation resembles the diffusion equation (1), in which the physical time $t$ is replaced by the boundary local time $\ell$, and the Laplace operator $\Delta$ is replaced by $-\mathcal{M}_{p}$. 
When the boundary $\partial \Omega$ of the domain is bounded, the Dirichlet-to-Neumann operator has a discrete spectrum, i.e., a countable set of positive eigenvalues $\mu_{n}^{(p)}$ and eigenfunctions $v_{n}^{(p)}(s)$ forming a complete orthonormal basis in the space $L_{2}(\partial \Omega)$ :

$$
\mathcal{M}_{p} v_{n}^{(p)}(s)=\mu_{n}^{(p)} v_{n}^{(p)}(s)
$$

The surface hopping propagator admits thus the spectral expansion:

$$
\Sigma_{p}\left(\boldsymbol{s}, \ell \mid \boldsymbol{s}_{0}\right)=\sum_{n}\left[v_{n}^{(p)}\left(\boldsymbol{s}_{0}\right)\right]^{*} v_{n}^{(p)}(\boldsymbol{s}) e^{-\mu_{n}^{(p)} \ell}
$$

where asterisk denotes complex conjugate. In other words, finding $\Sigma_{p}\left(s, \ell \mid s_{0}\right)$ is equivalent to studying the spectral properties of the Dirichlet-to-Neumann operator $\mathcal{M}_{p}$.

When $p>0$, all eigenvalues $\mu_{n}^{(p)}$ are strictly positive, and the surface hopping propagator vanishes as $\ell$ increases. This is a direct consequence of bulk reaction that may lead to eventual death or disappearance of the diffusing particle during its motion. In particular,

$$
\int_{\partial \Omega} d s \Sigma_{p}\left(s, \ell \mid s_{0}\right)<1 \quad(\ell>0),
$$

i.e., this density is not normalized to 1 , in the same way as the conventional propagator $G_{q}\left(\boldsymbol{x}, t \mid \boldsymbol{x}_{0}\right)$ is not normalized to 1 in the presence of reactive boundary $(q>0)$.

In turn, the case $p=0$ is more subtle. For restricted diffusion in a bounded domain, the ground eigenfunction is constant, $v_{0}^{(0)}(\boldsymbol{s})=|\partial \Omega|^{-1 / 2}$, whereas the associated eigenvalue is zero: $\mu_{0}^{(0)}=0$. Due to the orthogonality of other eigenfunctions to $v_{0}^{(0)}$, the surface hopping propagator is normalized to 1 :

$$
\int_{\partial \Omega} d s \Sigma_{0}\left(s, \ell \mid s_{0}\right)=1 \quad(\ell>0) .
$$

In contrast, if diffusion is transient, all eigenvalues $\mu_{n}^{(0)}$ are strictly positive, and the normalization is lost again, here, due to the possibility of escaping at infinity. This is the case of diffusion in the exterior of a bounded domain in $\mathbb{R}^{d}$ with $d \geq 3$ (for $d=2$, see a short discussion in Sec. A 4).

\section{Relation to other quantities}

As shown in [34], the surface hopping propagator opens the door to access most common diffusion-reaction characteristics such as the full propagator, the conventional propagator, the first-passage time distribution, and the reaction rate. In particular, the Laplace transform of the full propagator reads

$$
\begin{aligned}
& \tilde{P}\left(\boldsymbol{x}, \ell, p \mid \boldsymbol{x}_{0}\right)=\tilde{G}_{\infty}\left(\boldsymbol{x}, p \mid \boldsymbol{x}_{0}\right) \delta(\ell) \\
& +\int_{\partial \Omega} d \boldsymbol{s}_{0} \int_{\partial \Omega} d \boldsymbol{s} \tilde{j}_{\infty}(\boldsymbol{s}, p \mid \boldsymbol{x}) \frac{\Sigma_{p}\left(\boldsymbol{s}, \ell \mid \boldsymbol{s}_{0}\right)}{D} \tilde{j}_{\infty}\left(\boldsymbol{s}_{0}, p \mid \boldsymbol{x}_{0}\right),
\end{aligned}
$$

where $G_{\infty}\left(\boldsymbol{x}, t \mid \boldsymbol{x}_{0}\right)$ is the propagator for perfectly reactive boundary (with Dirichlet boundary condition $\left.G_{\infty}\left(\boldsymbol{x}, t \mid \boldsymbol{x}_{0}\right)_{\mid \partial \Omega}=0\right)$,

$$
j_{\infty}\left(\boldsymbol{s}, t \mid \boldsymbol{x}_{0}\right)=-D\left(\partial_{\boldsymbol{n}} G_{\infty}\left(\boldsymbol{x}, t \mid \boldsymbol{x}_{0}\right)\right)_{\boldsymbol{x}=\boldsymbol{s}}
$$

is the probability flux density on that boundary, and tilde denotes the Laplace transform with respect to time $t$, e.g.,

$$
\tilde{P}\left(\boldsymbol{x}, \ell, p \mid \boldsymbol{x}_{0}\right)=\int_{0}^{\infty} d t e^{-p t} P\left(\boldsymbol{x}, \ell, t \mid \boldsymbol{x}_{0}\right) .
$$

Substituting the spectral expansion (12) into Eq. (15), one also gets

$$
\begin{aligned}
\tilde{P}\left(\boldsymbol{x}, \ell, p \mid \boldsymbol{x}_{0}\right) & =\tilde{G}_{\infty}\left(\boldsymbol{x}, p \mid \boldsymbol{x}_{0}\right) \delta(\ell) \\
& +\frac{1}{D} \sum_{n}\left[V_{n}^{(p)}\left(\boldsymbol{x}_{0}\right)\right]^{*} V_{n}^{(p)}(\boldsymbol{x}) e^{-\mu_{n}^{(p)} \ell},
\end{aligned}
$$

with

$$
V_{n}^{(p)}(\boldsymbol{x})=\int_{\partial \Omega} d \boldsymbol{s} \tilde{j}_{\infty}(\boldsymbol{s}, p \mid \boldsymbol{x}) v_{n}^{(p)}(\boldsymbol{s}) .
$$

In turn, the full propagator determines most common quantities of diffusion-influenced reactions, in particular, the conventional propagator via Eq. (3). Moreover, one gets the marginal probability density of the boundary local time $\ell_{t}$ (see also $\left.[52,53]\right)$ :

$$
\rho\left(\ell, t \mid \boldsymbol{x}_{0}\right)=\int_{\Omega} d \boldsymbol{x} P\left(\boldsymbol{x}, \ell, t \mid \boldsymbol{x}_{0}\right),
$$

and the probability density of the first-crossing time $T_{\ell}=$ $\inf \left\{t>0: \ell_{t}>\ell\right\}$ of a level $\ell$ by $\ell_{t}$ :

$$
U\left(\ell, t \mid \boldsymbol{x}_{0}\right)=D \int_{\partial \Omega} d s P\left(s, \ell, t \mid \boldsymbol{x}_{0}\right) .
$$

The latter determines the probability density of the conventional first-passage time to a partial reactive boundary as:

$$
H_{q}\left(t \mid \boldsymbol{x}_{0}\right)=\int_{0}^{\infty} d \ell q e^{-q \ell} U\left(\ell, t \mid \boldsymbol{x}_{0}\right) .
$$

In the Laplace domain, one can use the spectral expansion (18) to write

$$
\tilde{U}\left(\ell, p \mid \boldsymbol{x}_{0}\right)=\sum_{n}\left[V_{n}^{(p)}\left(\boldsymbol{x}_{0}\right)\right]^{*} e^{-\mu_{n}^{(p)} \ell} \int_{\partial \Omega} d \boldsymbol{s} v_{n}^{(p)}(\boldsymbol{s}) .
$$


Inverting the Laplace transform, one gets $U\left(\ell, t \mid \boldsymbol{x}_{0}\right)$ and thus gains access via Eq. (22) to the whole family of probability densities $H_{q}\left(t \mid \boldsymbol{x}_{0}\right)$.

The surface hopping propagator also determines the spread harmonic measure density, $\omega_{q}\left(\boldsymbol{s} \mid \boldsymbol{x}_{0}\right)$, which characterizes the boundary point on a partially reactive boundary, at which reaction occurs [62-64]. For a particle started from $\boldsymbol{x}_{0}$, one has

$$
\omega_{q}\left(\boldsymbol{s} \mid \boldsymbol{x}_{0}\right)=\int_{\partial \Omega} d \boldsymbol{s}_{0} \omega_{q}\left(\boldsymbol{s} \mid \boldsymbol{s}_{0}\right) \tilde{j}_{\infty}\left(\boldsymbol{s}_{0}, 0 \mid \boldsymbol{x}_{0}\right),
$$

where

$$
\omega_{q}\left(s \mid s_{0}\right)=\int_{0}^{\infty} d \ell q e^{-q \ell} \Sigma_{0}\left(s, \ell \mid s_{0}\right) .
$$

More generally, the Laplace transform of $q \Sigma_{p}\left(\boldsymbol{s}, \ell \mid \boldsymbol{s}_{0}\right)$ with respect to the boundary local time $\ell$ yields the probability density of the reaction point $s$ on the boundary in the presence of bulk reactions with the rate $p$.

\section{Extension}

In the above construction of the surface hopping propagator $\Sigma_{p}\left(\boldsymbol{s}, \ell \mid \boldsymbol{s}_{0}\right)$ and the full propagator $P\left(\boldsymbol{x}, \ell, t \mid \boldsymbol{x}_{0}\right)$, the boundary local time $\ell$ is counted on the whole boundary $\partial \Omega$ of the confining domain $\Omega$. This boundary local time is then used to incorporate surface reactions, like in Eq. (3). In certain applications, however, only a subset of the boundary, $\Gamma \subset \partial \Omega$, is reactive, whereas the remaining part $\partial \Omega \backslash \Gamma$ is inert and just passively confines the diffusing particle inside the domain. This is a typical case of an escape through a hole $\Gamma$, or of a target $\Gamma$ surrounded by a reflecting surface. As encounters of the particle with the passive part of the boundary do not matter, one needs to count the boundary local time only on the reactive part $\Gamma$.

An extension to this setting is straightforward. In fact, one can re-define $\ell_{t}$ through the residence time in a close vicinity of the reactive part: $\Gamma_{a}=\{\boldsymbol{x} \in \Omega:|\boldsymbol{x}-\Gamma|<a\}$ :

$$
\ell_{t}=\lim _{a \rightarrow 0} \frac{D}{a} \int_{0}^{t} d t^{\prime} \mathbb{I}_{\Gamma_{a}}\left(\boldsymbol{X}_{t^{\prime}}\right) .
$$

The associated surface hopping propagator can be constructed as earlier by modifying the definition of the Dirichlet-to-Neumann operator. In fact, as the passive part of the boundary, $\partial \Omega \backslash \Gamma$, is irrelevant, one can define the Dirichlet-to-Neumann operator $\mathcal{M}_{p}^{\Gamma}$, acting on functions on $\Gamma$ as $\mathcal{M}_{p}^{\Gamma}: f \rightarrow g=\left(\partial_{\boldsymbol{n}} w\right)_{\mid \Gamma}$, where $w$ is the solution of the mixed Dirichlet-Neumann boundary value problem:

$$
(p-D \Delta) w=0 \quad \text { in } \Omega, \quad\left\{\begin{array}{l}
w_{\mid \Gamma}=f \\
\left(\partial_{\boldsymbol{n}} w\right)_{\mid \partial \Omega \backslash \Gamma}=0 .
\end{array}\right.
$$

Here, the Neumann boundary condition on $\partial \Omega \backslash \Gamma$ implements explicitly the reflecting character of the passive part of the boundary. In the following, we will present several examples of the surface hopping propagator for a subset of the boundary.

\section{EXPLICIT RESULTS FOR SEVERAL CONFINING DOMAINS}

In this section, we illustrate the properties of the surface hopping propagator and related quantities for several confining domains, for which the eigenbasis of the Dirichlet-to-Neumann operator can be explicitly derived. Even though these spectral properties are known to experts, we will provide some clarifications to guide readers. We start with the half-space, for which all quantities, including the full propagator, will be derived in closed explicit forms. Then we consider two-dimensional circular annuli between two concentric circles that include as limiting cases the interior and the exterior of a disk. An extension of these results to three-dimensional circular cylinders is briefly presented. Similarly, we discuss spherical shells between two concentric spheres that include the interior and the exterior of a ball. A numerical computation of the eigenmodes of the Dirichlet-to-Neumann operator in non-concentric perforated spherical domains is discussed in $[32,65]$. Technical derivations are moved to Appendices.

\section{A. Half-space}

It is instructive to start with the case of the half-space $\Omega=\left\{\boldsymbol{x}=\left(x_{1}, \ldots, x_{d}\right) \in \mathbb{R}^{d}: x_{d}>0\right\}$, for which all quantities of interest can be obtained in a closed analytic form. Even though the boundary $\partial \Omega$ of the half-space is not bounded, the derived formulas can be adapted. In this case, the spectrum of the Dirichlet-toNeumann operator is continuous, and sums over eigenmodes should be replaced by integrals. Moreover, the solutions of the eigenvalue equation $(11), v_{n}^{(p)}$, are not $L_{2}(\partial \Omega)$-normalized and thus cannot be called "eigenfunctions". Nevertheless, we will keep this term for $v_{n}^{(p)}$, bearing in mind its limitations.

To clarify the ideas, we start again with the planar case $(d=2)$. The translational symmetry of the boundary implies that $v_{n}(s)=e^{i n s} / \sqrt{2 \pi}$ are the "eigenfunctions" of the Dirichlet-to-Neumann operator $\mathcal{M}_{p}$. In fact, since $w(x, y)=e^{i n x-y \sqrt{n^{2}+p / D}}$ satisfies the modified Helmholtz equation (9) in the upper half-plane, $e^{i n s}$ is an "eigenfunction", associated to the "eigenvalue" $\mu_{n}^{(p)}=\sqrt{n^{2}+p / D}$. Note that this "eigenfunction" does not depend on $p$ due to the above symmetry. The prefactor $1 / \sqrt{2 \pi}$ comes from the orthogonality of "eigenfunc- 
tions":

$$
\int_{-\infty}^{\infty} d s v_{n}(s)\left[v_{n^{\prime}}(s)\right]^{*}=\delta\left(n-n^{\prime}\right) .
$$

Skipping technical details, we formally rewrite the spectral expansion (12) of the surface hopping propagator as

$$
\Sigma_{p}\left(s, \ell \mid s_{0}\right)=\int_{-\infty}^{\infty} d n\left[v_{n}\left(s_{0}\right)\right]^{*} v_{n}(s) \exp \left(-\mu_{n}^{(p)} \ell\right),
$$

where the former summation index $n$ now takes real values in $\mathbb{R}$. As a consequence, we get

$$
\begin{aligned}
\Sigma_{p}\left(s, \ell \mid s_{0}\right) & =\int_{-\infty}^{\infty} \frac{d n}{2 \pi} e^{i n\left(s_{0}-s\right)-\ell \sqrt{n^{2}+p / D}} \\
& =\frac{\ell}{\pi\left(\ell^{2}+\left(s-s_{0}\right)^{2}\right)} \zeta K_{1}(\zeta),
\end{aligned}
$$

with

$$
\zeta=\sqrt{p / D} \sqrt{\ell^{2}+\left(s-s_{0}\right)^{2}},
$$

and $K_{\nu}(z)$ is the modified Bessel function of the second kind. At $p=0$, we retrieve the Cauchy density (8). In this case, the surface exploration up to the boundary local time $\ell$ is equivalent to the first arrival onto that surface of Brownian motion started from $\left(s_{0}, \ell\right)$, i.e. the distance $\ell$ above the surface. Figure 2 illustrates the behavior of the surface hopping propagator. Changing progressively the boundary local time $\ell$, one observes the spreading of the surface hopping propagator.

For "immortal" particles $(p=0)$, the surface hopping propagator exhibits heavy tails, $\Sigma_{0}\left(s, \ell \mid s_{0}\right) \propto\left|s-s_{0}\right|^{-2}$, in particular, the variance of the arrival point $s$ is infinite (the mean is $s_{0}$ due to the symmetric form of this propagator). Such displacements with infinite variance resemble Lévy flights [66]. This is the consequence of unbounded exploration region that allows for very long and far-reaching trajectories. The situation is drastically different for "mortal" particles $(p>0)$, for which long trajectories are penalized by tiny chances of survival. In fact, the central part of this distribution (when $\ell^{2}+\left|s-s_{0}\right|^{2} \ll$ $D / p$ ) resembles again the Cauchy density (Fig. 2(b)), which is however truncated by exponential tails at large $s($ when $\zeta \gg 1): \Sigma_{p}\left(s, \ell \mid s_{0}\right) \propto e^{-\zeta} \sim e^{-\left|s-s_{0}\right| \sqrt{p / D}}$. The bulk rate $p$ (or, more precisely, $p / D$ ) controls this truncation. As a consequence, all the positive moments of the arrival boundary point are finite. Expectedly, the surface hopping propagator is not normalized to 1 for $p>0$ :

$$
\int_{\mathbb{R}} d s \Sigma_{p}\left(s, \ell \mid s_{0}\right)=e^{-\ell \sqrt{p / D}} .
$$

Interestingly, even the conditional surface hopping propagator that accounts only for the survived particles after
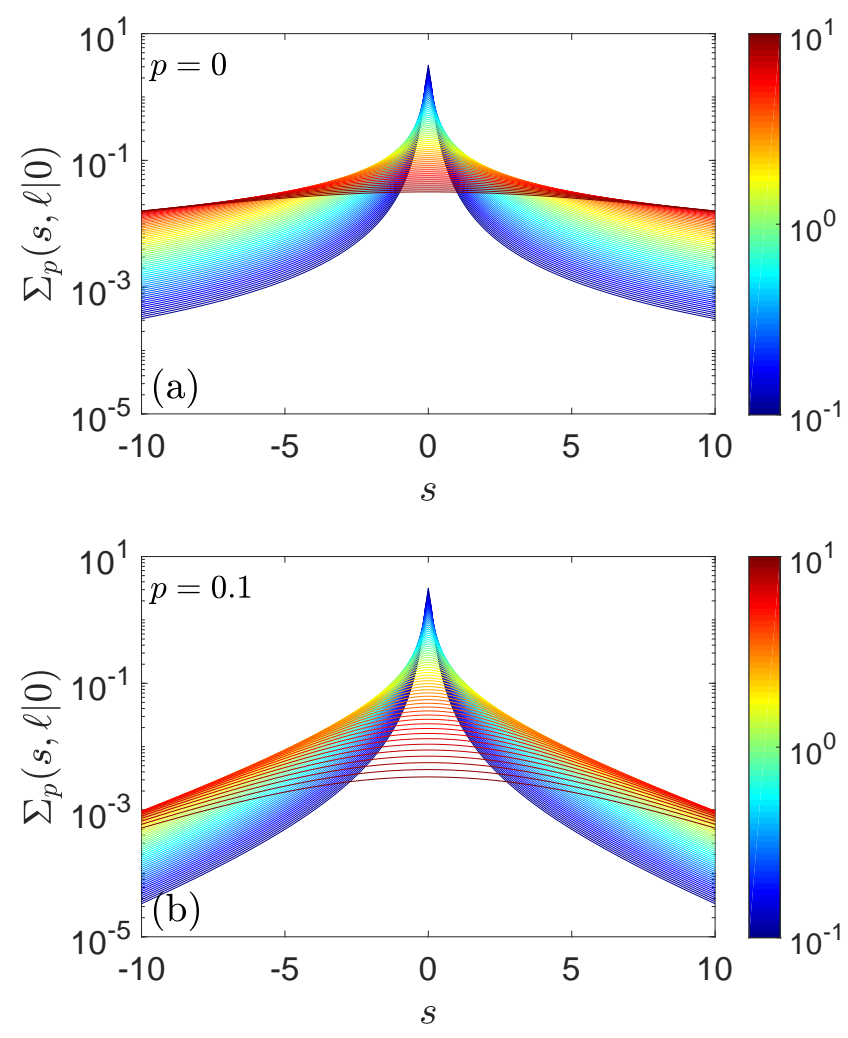

FIG. 2: The surface hopping propagator $\Sigma_{p}(s, \ell \mid 0)$, given by Eq. (30), for diffusion in the upper half-plane for $p=0$ (a) and $p=0.1(\mathbf{b})$, and 64 values of $\ell$, logarithmically spaced in the range from $10^{-1}$ (blue curves) to $10^{1}$ (red curves), with $D=1$.

renormalization by $e^{-\ell \sqrt{p / D}}$, shows an exponential decay with $s$.

In higher dimensions, the "eigenfunctions" and "eigenvalues" of the Dirichlet-to-Neumann operator have similar form:

$$
v_{\boldsymbol{n}}(\boldsymbol{s})=\frac{e^{i(\boldsymbol{n} \cdot \boldsymbol{s})}}{(2 \pi)^{(d-1) / 2}}, \quad \mu_{\boldsymbol{n}}^{(p)}=\sqrt{|\boldsymbol{n}|^{2}+p / D},
$$

with the "multi-index" $\boldsymbol{n}=\left(n_{1}, \ldots, n_{d-1}\right) \in \mathbb{R}^{d-1}$. The surface propagator can thus be written as

$$
\Sigma_{p}\left(\boldsymbol{s}, \ell \mid \boldsymbol{s}_{0}\right)=\int_{\mathbb{R}^{d-1}} \frac{d \boldsymbol{n}}{(2 \pi)^{d-1}} e^{i \boldsymbol{n} \cdot\left(\boldsymbol{s}_{0}-\boldsymbol{s}\right)-\ell \sqrt{|\boldsymbol{n}|^{2}+p / D}} .
$$

In spherical coordinates, the integral over all orientations gives

$$
\begin{aligned}
\Sigma_{p}\left(\boldsymbol{s}, \ell \mid \boldsymbol{s}_{0}\right) & =\frac{\left|\boldsymbol{s}-\boldsymbol{s}_{0}\right|^{\frac{3-d}{2}}}{(2 \pi)^{\frac{d-1}{2}}} \int_{0}^{\infty} d n n^{\frac{d-1}{2}} J_{\frac{d-3}{2}}\left(n\left|s-s_{0}\right|\right) \\
& \times e^{-\ell \sqrt{n^{2}+p / D}} \\
& =\Sigma_{0}\left(\boldsymbol{s}, \ell \mid \boldsymbol{s}_{0}\right) \frac{\zeta^{\frac{d}{2}} K_{\frac{d}{2}}(\zeta)}{\Gamma\left(\frac{d}{2}\right) 2^{\frac{d}{2}-1}}
\end{aligned}
$$


where $\zeta$ is given by Eq. (31), and

$$
\Sigma_{0}\left(\boldsymbol{s}, \ell \mid \boldsymbol{s}_{0}\right)=\frac{\Gamma(d / 2)}{\pi^{d / 2}} \frac{\ell}{\left(\ell^{2}+\left|s-s_{0}\right|^{2}\right)^{d / 2}}
$$

is again the harmonic measure density on the hyperplane $\mathbb{R}^{d-1}$ (see also [64]), and $J_{\nu}(z)$ is the Bessel function of the first kind. In the right-hand side of Eq. (35), one can recognize the Laplace-transformed probability flux density $\tilde{j}_{\infty}\left(s, p \mid\left(s_{0}, \ell\right)\right)$ onto a perfectly absorbing hyperplane from the bulk point $\boldsymbol{x}_{0}=\left(\boldsymbol{s}_{0}, \ell\right)$. The inverse Laplace transform with respect to $p$ yields then

$$
\begin{aligned}
\mathcal{L}_{t}^{-1}\left\{\Sigma_{p}\left(\boldsymbol{s}, \ell \mid \boldsymbol{s}_{0}\right)\right\} & =j_{\infty}\left(\boldsymbol{s}, t \mid\left(\boldsymbol{s}_{0}, \ell\right)\right) \\
& =\frac{\exp \left(-\frac{\left|\boldsymbol{s}-\boldsymbol{s}_{0}\right|^{2}}{4 D t}\right)}{(4 \pi D t)^{(d-1) / 2}} \frac{\ell \exp \left(-\frac{\ell^{2}}{4 D t}\right)}{\sqrt{4 \pi D t^{3}}} .
\end{aligned}
$$

We emphasize that this relation is specific to the case of the half-space.

Using this relation and the representation (34), one can easily compute the double integral over $s_{1}$ and $s_{2}$ in Eq. (18) to get

$$
\tilde{P}\left(\boldsymbol{x}, \ell, p \mid \boldsymbol{x}_{0}\right)=\tilde{G}_{\infty}\left(\boldsymbol{x}, p \mid \boldsymbol{x}_{0}\right) \delta(\ell)+\frac{\Sigma_{p}\left(\boldsymbol{y}, z+z_{0}+\ell \mid \boldsymbol{y}_{0}\right)}{D},
$$

where $\boldsymbol{x}=(\boldsymbol{y}, z)$ and $\boldsymbol{x}_{0}=\left(\boldsymbol{y}_{0}, z_{0}\right)$. The inverse Laplace transform with respect to $p$ yields then

$$
\begin{aligned}
& P\left(\boldsymbol{x}, \ell, t \mid \boldsymbol{x}_{0}\right)=G_{\infty}\left(\boldsymbol{x}, t \mid \boldsymbol{x}_{0}\right) \delta(\ell)+\frac{j_{\infty}\left(\boldsymbol{y}, t \mid\left(\boldsymbol{y}_{0}, z+z_{0}+\ell\right)\right)}{D} \\
& =\frac{\exp \left(-\frac{\left|\boldsymbol{y}-\boldsymbol{y}_{0}\right|^{2}}{4 D t}\right)}{(4 \pi D t)^{d / 2}}\left\{\left(e^{-\left(z-z_{0}\right)^{2} /(4 D t)}-e^{-\left(z+z_{0}\right)^{2} /(4 D t)}\right) \delta(\ell)\right. \\
& \left.+\frac{\ell+z+z_{0}}{D t} e^{-\left(z+z_{0}+\ell\right)^{2} /(4 D t)}\right\} .
\end{aligned}
$$

This is the explicit exact form of the full propagator for the half-space.

One can easily check that the integral over $\boldsymbol{x} \in \mathbb{R}_{+}^{d}$ gives the marginal probability density of the boundary local time $\ell_{t}$ :

$$
\rho\left(\ell, t \mid \boldsymbol{x}_{0}\right)=\operatorname{erf}\left(\frac{z_{0}}{\sqrt{4 D t}}\right) \delta(\ell)+\frac{\exp \left(-\frac{\left(z_{0}+\ell\right)^{2}}{4 D t}\right)}{\sqrt{\pi D t}} .
$$

This expression does not depend on the dimension $d$ and the lateral coordinate $\boldsymbol{y}_{0}$ of the starting point $\boldsymbol{x}_{0}$, given that the boundary local time is independent of lateral displacements and determined by the transverse motion (on the half-line). The distribution of the boundary local time $\ell_{t}$ was studied in $[52,53]$.

In turn, the integral of Eq. (39) over $\ell \in \mathbb{R}_{+}$yields the marginal probability density of the position, i.e., conventional propagator $G_{0}\left(\boldsymbol{x}, t \mid \boldsymbol{x}_{0}\right)$ in the half-space with reflecting boundary:

$$
\begin{aligned}
G_{0}\left(\boldsymbol{x}, t \mid \boldsymbol{x}_{0}\right) & =\frac{\exp \left(-\frac{\left|\boldsymbol{y}-\boldsymbol{y}_{0}\right|^{2}}{4 D t}\right)}{(4 \pi D t)^{d / 2}} \\
& \times\left(e^{-\left(z-z_{0}\right)^{2} /(4 D t)}+e^{-\left(z+z_{0}\right)^{2} /(4 D t)}\right) .
\end{aligned}
$$

Moreover, with the general expression (3), one retrieves the propagator $G_{q}\left(\boldsymbol{x}, t \mid \boldsymbol{x}_{0}\right)$ for reactive boundary:

$$
\begin{aligned}
& G_{q}\left(\boldsymbol{x}, t \mid \boldsymbol{x}_{0}\right)=\frac{\exp \left(-\frac{\left|\boldsymbol{y}-\boldsymbol{y}_{0}\right|^{2}}{4 D t}\right)}{(4 \pi D t)^{d / 2}}\left\{e^{-\left(z-z_{0}\right)^{2} /(4 D t)}+\right. \\
& \left.e^{-\left(z+z_{0}\right)^{2} /(4 D t)}\left(1-2 q \sqrt{\pi D t} \operatorname{erfcx}\left(\frac{z+z_{0}}{\sqrt{4 D t}}+q \sqrt{D t}\right)\right)\right\},
\end{aligned}
$$

where $\operatorname{erfcx}(z)=e^{z^{2}} \operatorname{erfc}(z)$ is the scaled complementary error function. Expectedly, the propagators in Eqs. (41, 42) exhibit translational invariance along $\boldsymbol{y}$ coordinate and are factored into the lateral Gaussian (free) propagator and the transverse propagator on the half-line $(0, \infty)$. Note that its integral over the arrival point $\boldsymbol{x}$ yields the survival probability

$S_{q}\left(t \mid \boldsymbol{x}_{0}\right)=\operatorname{erf}\left(\frac{z_{0}}{\sqrt{4 D t}}\right)+e^{-\frac{z_{0}^{2}}{4 D t}} \operatorname{erfcx}\left(\frac{z_{0}}{\sqrt{4 D t}}+q \sqrt{D t}\right)$

which does not depend on $\boldsymbol{y}_{0}$ and coincides with the survival probability for the semi-axis with the partially reactive endpoint. The classical expression for the associated probability density of the reaction time is then retrieved:

$$
\begin{aligned}
H_{q}\left(t \mid \boldsymbol{x}_{0}\right) & =q D e^{-z_{0}^{2} /(4 D t)}\left\{\frac{1}{\sqrt{\pi D t}}\right. \\
& \left.-q \operatorname{erfcx}\left(\frac{z_{0}}{\sqrt{4 D t}}+q \sqrt{D t}\right)\right\} .
\end{aligned}
$$

Finally, Eq. (21) yields

$$
U\left(\ell, t \mid \boldsymbol{x}_{0}\right)=\frac{\left(\ell+z_{0}\right) e^{-\left(\ell+z_{0}\right)^{2} /(4 D t)}}{\sqrt{4 \pi D t^{3}}},
$$

i.e., we retrieved the classical formula for the probability density of the first crossing time of a level $\ell$ by the boundary local time of reflected Brownian motion on the half-line (see, e.g., [54]).

To complete this section, we briefly mention that the above computations can be easily extended to a slab domain between parallel hyperplanes, one of which is reflecting (see [67] for more details). In other words, one can consider $\Omega=\left\{\boldsymbol{x} \in \mathbb{R}^{d}: 0<x_{d}<L\right\}$ and study the Dirichlet-to-Neumann operator on the hyperplane $x_{d}=0$ in the presence of the reflecting hyperplane $x_{d}=L$. The "eigenfunctions" of such Dirichlet-to-Neumann operator remain unchanged due to the translational symmetry, whereas the "eigenvalues" are

$$
\mu_{\boldsymbol{n}}^{(p)}=\sqrt{|\boldsymbol{n}|^{2}+p / D} \tanh \left(L \sqrt{|\boldsymbol{n}|^{2}+p / D}\right) .
$$

As $L \rightarrow \infty$, one retrieves the former case of the halfspace. The former integral representations for the surface hopping propagator and related quantities remain valid if Eq. (46) is used for $\mu_{\boldsymbol{n}}^{(p)}$. In contrast, the presence of $\tanh (z)$ prevents from getting simple closed formulas for these quantities in the case of a slab. 

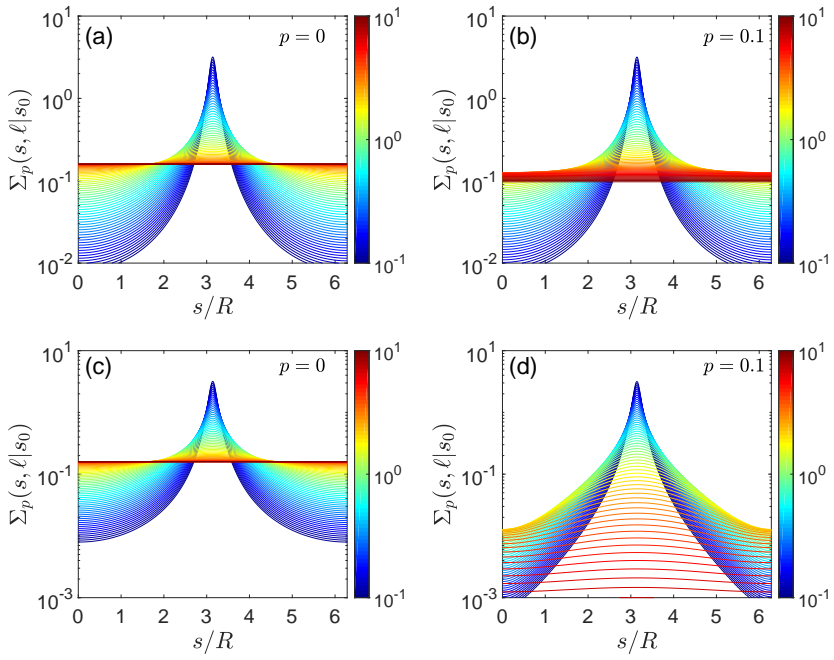

FIG. 3: (a,b) The surface hopping propagator $\Sigma_{p}\left(s, \ell \mid s_{0}\right)$, given by Eq. (A6), for diffusion inside a disk of radius $R$, for $p=0$ (a) and $p=0.1$ (b), and 64 values of $\ell$, logarithmically spaced in the range from $10^{-1}$ (blue curves) to $10^{1}$ (red curves), with $D=1$ and $s_{0} / R=\pi$. (c,d) The surface hopping propagator $\Sigma_{p}\left(s, \ell \mid s_{0}\right)$ for diffusion outside a disk of radius $R$, with the same parameters. Note that the propagators on panels (a) and (c) are identical but the vertical axis is cut differently. The series in Eq. (A6) is truncated above $|n|=100$.

\section{B. Circular annuli and spherical shells}

In Appendices A and B, we provide explicit formulas for the eigenfunctions and eigenvalues of the Dirichlet-toNeumann operator in several rotationally invariant domains: circular annuli, the interior and the exterior of a disk, circular cylinders, spherical shells, the interior and the exterior of a ball. These formulas allow one to get the surface hopping propagator $\Sigma_{p}\left(s, \ell \mid s_{0}\right)$ via the spectral expansion (12), as well as the full propagator $P\left(\boldsymbol{x}, \ell, t \mid \boldsymbol{x}_{0}\right)$ and all the related quantities, as discussed in Sec. II C. From these basic results, one can thoroughly investigate various diffusion-mediated surface phenomena in the above domains. In this paper, we keep our focus on the surface hopping propagator and illustrate its properties for these domains.

Figure $3(\mathrm{a}, \mathrm{b})$ shows the surface hopping propagator for the interior of a disk of radius $R$. At $p=0$ (panel (a)), the surface hopping propagator $\Sigma_{0}\left(s, \ell \mid s_{0}\right)$ coincides with the harmonic measure density on the circle, see Eq. (A14). Expectedly, it evolves from the Dirac distribution at $\ell=0$ to the uniform distribution $1 /(2 \pi R)$ as $\ell \rightarrow \infty$. When $p>0$ (panel (b)), eventual death of the particle during its bulk explorations affects this propagator. At small $\ell$, the particle spends short time in the bulk so that there is almost no effect of the bulk rate $p$ (at moderate $p)$ : blue curves on (a) and (b) panels are almost identical. As $\ell$ increases, the effect of $p$ becomes more prominent.
At large $\ell$, the particle has enough time to explore the interior of the disk, leading again to the uniform distribution of the arrival point. This can also be seen from the spectral expansion (12), in which the contribution from higher-order eigenmodes of the Dirichlet-to-Neumann operator vanished. The surface hopping propagator becomes almost flat again, $\Sigma_{p}\left(s, \ell \mid s_{0}\right) \simeq e^{-\mu_{0}^{(p)} \ell} /(2 \pi R)$, but its level is now attenuated by bulk reactions. As $\mu_{0}^{(p=0.1)} \approx 0.0494$, the attenuation factor $e^{-\mu_{0}^{(p)} \ell}$ is not strong even at $\ell=10$ in the considered example. However, one can still see a qualitative difference between $p=0$ and $p=0.1$ cases: in the former case, the curves approach the limit $1 /(2 \pi R)$, whereas in the latter case, they are progressively shifted downward.

Figure 3(c,d) shows the surface hopping propagator for the exterior of a disk of radius $R$. We note that Eq. (A14) for the surface hopping propagator at $p=0$ remains the same for diffusion outside the disk. This reflects the conformal invariance of the harmonic measure with respect to an inversion mapping of the interior of the disk to its exterior. In contrast, for $p>0$, the behavior of the surface hopping propagator is different for diffusion inside and outside the disk, especially for large $\ell$. In fact, the particle diffusing outside the disk undertakes much longer bulk excursions between successive encounters with the boundary and thus has higher chances to be killed by a bulk reaction. This leads to much smaller values of the surface hopping propagator (we recall that the propagator is not normalized to 1 for $p>0$ ).

Figure 4 presents the surface hopping propagator $\Sigma_{0}\left(s, \ell \mid s_{0}\right)$ for a circular annulus with both reactive circles of radii $R$ and $L$. As the boundary is composed of two circles, there are two distinct choices of the starting point $s_{0}$ (shown by red dot): either on the inner circle of radius $R$, or on the outer circle of radius $L$ (and one can set $s_{0}=0$ in both cases due to rotational invariance). For convenience of presentation, the curvilinear coordinate $s$ runs here from $-2 \pi R$ to 0 for the inner circle, and from 0 to $2 \pi L$ for the outer circle. On the horizontal axis, $s$ is rescaled by $R$ for negative values and by $L$ for positive values so that the horizontal axis varies from $-2 \pi$ and $2 \pi$. Let us first consider the particle started on the inner circle (Fig. 4(a)). At small $\ell$ (blue curves), this particle does not move far away from the starting point $s_{0}$, so that $\Sigma_{0}\left(s, \ell \mid s_{0}\right)$ rapidly decays when $s / R$ varies from 0 to $-\pi$ (its later increase for $s / R$ ranging from $-\pi$ to $-2 \pi$ is due to the symmetry). Similarly, bulk excursions of the particle rarely terminate at the outer circle so that the surface hopping propagator remains small on that boundary (the range of positive $s$ ). Clearly, the minimum corresponds to the boundary point $s=\pi L$ on the outer boundary which is located behind the starting point $s_{0}$. As the boundary local time increases, the particle explores further boundary regions, both on the inner and outer circles. In the limit $\ell \rightarrow \infty$, the surface hopping propagator approaches the uniform density, $1 /(2 \pi(R+L))$, on both circles, as expected. When the starting point is on the outer circle (Fig. 4(b)), the picture is very similar, i.e., the particle 

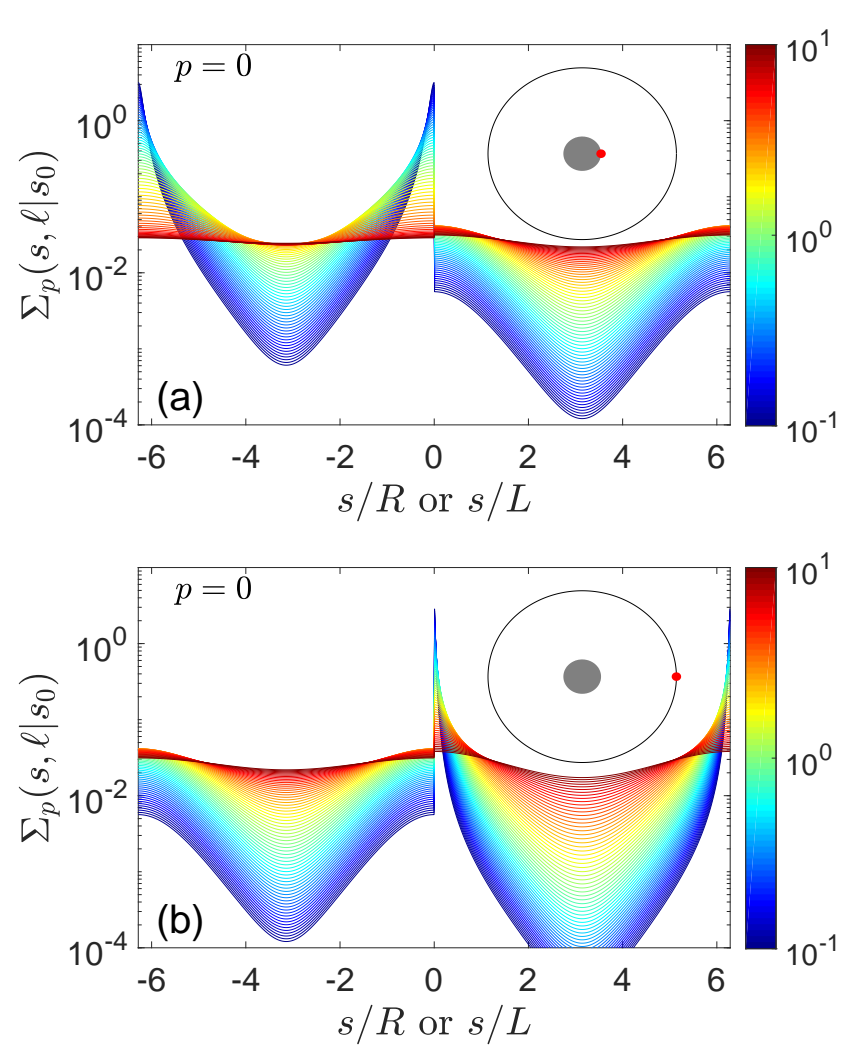

FIG. 4: The surface hopping propagator $\Sigma_{p}\left(s, \ell \mid s_{0}\right)$ for a circular annulus with both reactive circles of radii $R$ and $L$, for 64 values of $\ell$, logarithmically spaced in the range from $10^{-1}$ (blue curves) to $10^{1}$ (red curves), with $p=0, L=5 R$, $D=1$. (a) The starting point $s_{0}=0$ (red dot) is on the inner circle; (b) the starting point $s_{0}=0$ is on the outer circle. The series is truncated above $|n|=1000$.

remains on the outer circle (close to the starting point) at small $\ell$ but then spreads away. Note that the stronger decay of the surface hopping propagator is caused by the fact that the outer circle is much longer than the inner one. For this reason, a large truncation order was needed to accurately compute $\Sigma_{0}\left(s, \ell \mid s_{0}\right)$ in this case.

For "mortal" particles (not shown), eventual death due to the bulk rate $p>0$ penalizes long trajectories, as in the case of diffusion inside a disk. Moreover, in highly reactive media, the particle has tiny chances to move from one circle to the other, and these parts of the boundary become decoupled. In other words, as there is almost no survived particles that crossed the annulus, the properties of the boundary far away from the starting point do not matter.

Figure 5 shows similar results for the interior and exterior of a ball. As previously, $\Sigma_{p}\left(s, \ell \mid s_{0}\right)$ behaves differently for diffusion inside and outside the ball. However, this difference is considerably enhanced in three dimensions due to the recurrent versus transient character of diffusion. In fact, the particle diffusing outside a ball can escape to infinity with a finite probability. As a conse-
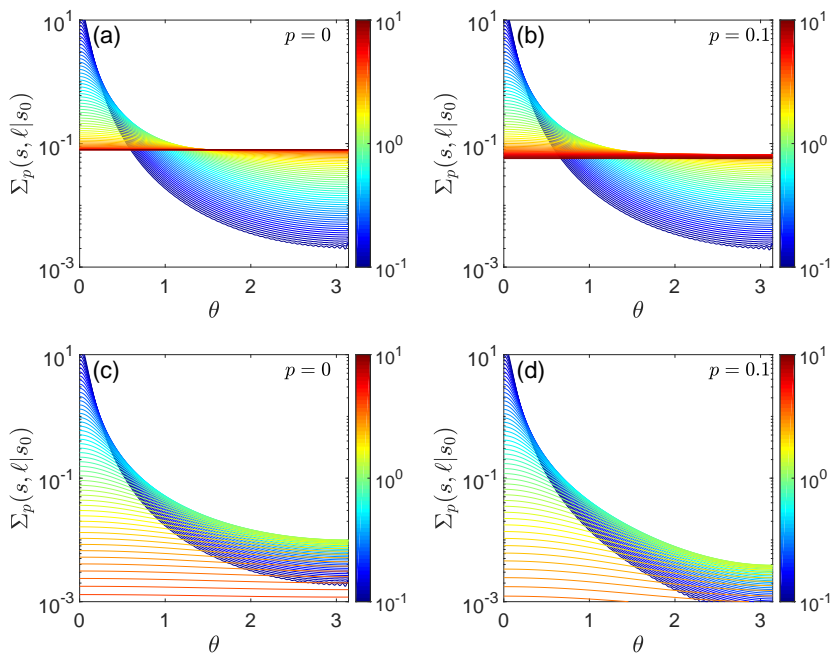

FIG. 5: (a,b) The surface hopping propagator $\Sigma_{p}\left(\boldsymbol{s}, \ell \mid \boldsymbol{s}_{0}\right)$, given by Eq. (B22), for diffusion inside a ball of radius $R$, for $p=0$ (a) and $p=0.1$ (b), and 64 values of $\ell$, logarithmically spaced in the range from $10^{-1}$ (blue curves) to $10^{1}$ (red curves), with $D=1, \boldsymbol{s}_{0}=(0,0, R)$ (the North pole), and $\boldsymbol{s}=(R \sin \theta, 0, R \cos \theta)$ (i.e., $\theta$ is the angle between $\boldsymbol{s}_{0}$ and $\boldsymbol{s})$. $(\mathbf{c}, \mathbf{d})$ The surface hopping propagator $\Sigma_{p}\left(\boldsymbol{s}, \ell \mid \boldsymbol{s}_{0}\right)$ for diffusion outside a ball of radius $R$, with the same parameters. The series in Eq. (B22) is truncated above $n=100$ that results in small oscillations seen for blue curves.

quence, the surface hopping propagator is not normalized to 1 here even for $p=0$. Actually, the normalization constant is $e^{-\ell / R}$, i.e., the escape probability is $1-e^{-\ell / R}$. On panel (c), one can see that the surface hopping propagator becomes again uniform (as the contribution of higher-order eigenfunctions vanishes) but attenuated by the factor $e^{-\ell / R}$.

Finally, Fig. 6 illustrates the surface hopping propagator on a spherical target of radius $R$, surrounded by an outer reflecting sphere of radius $L$. This setting is qualitatively in between the interior and the exterior of a ball. On one hand, as this domain is bounded and diffusion is recurrent, the surface hopping propagator evolves towards the uniform density $1 /\left(4 \pi R^{2}\right)$, as for the interior case. On the other hand, the outer reflecting sphere is located relatively far from the target and thus allows for long trajectories, as for the exterior case. For $p=0$, the panel (a) of Fig. 6 resembles the panel (a) of Fig. 5 , even so diffusion occurs in different regions in these two settings. In contrast, when $p=0.1$, the panel (b) of Fig. 6 is much closer to the panel (d) of Fig. 5. In fact, in both cases, the particle is allowed to undertake long trajectories between successive encounters with the target. 

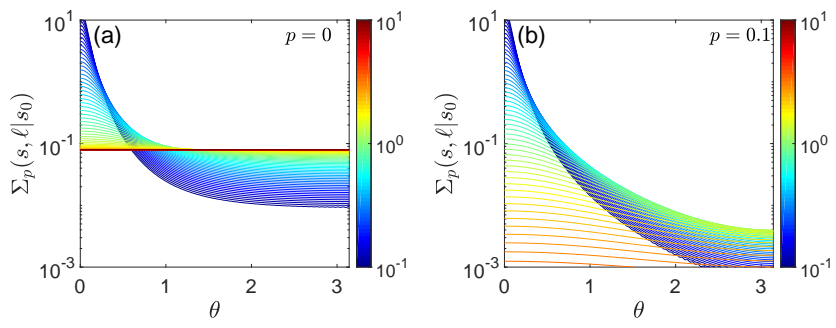

FIG. 6: The surface hopping propagator $\Sigma_{p}\left(\boldsymbol{s}, \ell \mid \boldsymbol{s}_{0}\right)$, given by Eq. (B6), on a spherical target of radius $R$, surrounded by an outer reflecting sphere of radius $L$, for $p=0$ (a) and $p=0.1$ (b), and 64 values of $\ell$, logarithmically spaced in the range from $10^{-1}$ (blue curves) to $10^{1}$ (red curves), with $L=10 R, D=1, \boldsymbol{s}_{0}=(0,0, R)$ (the North pole), and $\boldsymbol{s}=$ $(R \sin \theta, 0, R \cos \theta)$ (i.e., $\theta$ is the angle between $\boldsymbol{s}_{0}$ and $\left.\boldsymbol{s}\right)$. The series in Eq. (B6) is truncated above $n=100$.

\section{DISCUSSION AND CONCLUSION}

In this paper, we investigated the properties of the surface hopping propagator $\Sigma_{p}\left(\boldsymbol{s}, \ell \mid \boldsymbol{s}_{0}\right)$ recently introduced in [34]. This is a conceptually new quantity that describes bulk-diffusion-mediated exploration of a surface. In contrast to former works [46-50], which relied on coupled bulk-surface diffusion equations and aimed to characterize the position of the particle on a surface after some physical time $t$, here we operate with the boundary local time $\ell$, which is a proxy of the number of encounters with that surface. The surface hopping propagator turns out to be dual to the conventional propagator $G_{q}\left(\boldsymbol{x}, t \mid \boldsymbol{x}_{0}\right)$. In fact, as $G_{q}\left(\boldsymbol{x}, t \mid \boldsymbol{x}_{0}\right)$ characterizes displacements between bulk points $\boldsymbol{x}_{0}$ and $\boldsymbol{x}$ in physical time $t$ (i.e., after a number of bulk jumps), $\Sigma_{p}\left(\boldsymbol{s}, \ell \mid \boldsymbol{s}_{0}\right)$ characterizes effective displacements between boundary points $\boldsymbol{s}_{0}$ and $\boldsymbol{s}$ in boundary local time $\ell$ (i.e., after a number of reflections on the boundary). While $G_{q}\left(\boldsymbol{x}, t \mid \boldsymbol{x}_{0}\right)$ is the semi-group of the Laplace operator $-\Delta$ (acting in the bulk), $\Sigma_{p}\left(\boldsymbol{s}, \ell \mid \boldsymbol{s}_{0}\right)$ is the semi-group of the Dirichlet-to-Neumann operator $\mathcal{M}_{p}$ (acting on the boundary). In this light, the spectral expansion (12) is dual to the spectral expansion for the conventional propagator:

$$
G_{q}\left(\boldsymbol{x}, t \mid \boldsymbol{x}_{0}\right)=\sum_{n}\left[u_{n}^{(q)}\left(\boldsymbol{x}_{0}\right)\right]^{*} u_{n}^{(q)}(\boldsymbol{x}) e^{-\lambda_{n}^{(q)} t},
$$

where $\lambda_{n}^{(q)}$ and $u_{n}^{(q)}(\boldsymbol{x})$ are the eigenvalues and $L_{2}(\Omega)$ normalized eigenfunctions of the diffusion operator $-D \Delta$ :

$$
\begin{aligned}
-D \Delta u_{n}^{(q)} & =\lambda_{n}^{(q)} u_{n}^{(q)} \quad(\boldsymbol{x} \in \Omega), \\
\partial_{\boldsymbol{n}} u_{n}^{(q)}+q u_{n}^{(q)} & =0 \quad(\boldsymbol{x} \in \partial \Omega),
\end{aligned}
$$

where we highlighted the dependence on the reactivity parameter $q$ through the Robin boundary condition (48b). Similarity and duality of Eqs. $(12,47)$ are remarkable. We recall that the spectral expansion (47) is valid for a bounded domain $\Omega$, whereas the spectral expansion (12) is valid for a bounded boundary $\partial \Omega$. As a consequence, Eq. (12) seems to be more general, as it is also valid when $\Omega$ is the exterior of a bounded domain, for which Eq. (47) is not applicable anymore. In spite of this considerable advantage, the Dirichletto-Neumann operator and its eigenbasis were not earlier employed to describe diffusion-influenced reactions and other diffusion-mediated surface phenomena. The present paper, along with Refs. [34, 68], aim to shift the theoretical description of these phenomena towards a new fundamental ground. Using the formulas derived in this paper, one can access directly not only the surface hopping propagator, but all the related quantities, including the full propagator, first-passage time distribution, the survival probability and the reaction rate.

\section{Acknowledgments}

The author acknowledges a partial financial support from the Alexander von Humboldt Foundation through a Bessel Research Award.

\section{Appendix A: Circular annulus}

Here, we consider a circular annulus between two concentric circles of radii $R<L: \Omega=\left\{\boldsymbol{x} \in \mathbb{R}^{2}: R<|\boldsymbol{x}|<\right.$ $L\}$. There are four possible combinations of surface reactivity: (i) both circles are reactive, (ii) the inner circle is reflecting while the outer circle is reactive, (iii) the inner circle is reactive while the outer circle is reflecting, and (iv) both circles are reflecting. As surface reaction is not possible in the last case, it is excluded. The first case corresponds directly to our general setting when the whole boundary is reactive. However, as this case involves two disjoint parts of the boundary (the inner and the outer circles), its analysis is the most complicated. For this reason, we start with the case (ii), then briefly discuss the case (iii), and finally give the solution for the case (i).

\section{Reactive outer circle}

In order to determine the spectrum of the Dirichlet-toNeumann operator associated with the outer circle $\Gamma=$ $\left\{\boldsymbol{x} \in \mathbb{R}^{2} \quad:|\boldsymbol{x}|=L\right\}$, one needs to solve the mixed boundary value problem (27). Its general solution can be searched in polar coordinates $(r, \phi)$ as:

$$
w(r, \phi)=\sum_{n=-\infty}^{\infty} c_{n} e^{i n \phi} g_{n}(r),
$$


where $c_{n}$ are unknown coefficients (to be fixed by the boundary condition),

$$
g_{n}(r)=\frac{K_{n}^{\prime}(\alpha R) I_{n}(\alpha r)-I_{n}^{\prime}(\alpha R) K_{n}(\alpha r)}{K_{n}^{\prime}(\alpha R) I_{n}(\alpha L)-I_{n}^{\prime}(\alpha R) K_{n}(\alpha L)} .
$$

are radial functions with $\alpha=\sqrt{p / D}$, prime denotes the derivative with respect to the argument, and $I_{n}(z)$ is the modified Bessel functions of the first kind. One can easily check that $g_{n}^{\prime}(R)=0$ by construction. For convenience, we have chosen a particular normalization $g_{n}(L)=1$. As the normal derivative is equal to the radial derivative, the action of the Dirichlet-to-Neumann operator $\mathcal{M}_{p}$ does not affect the angular part. In other words, the rotational symmetry of this domain implies that Fourier harmonics are the eigenfunctions of $\mathcal{M}_{p}$, defined on the outer circle $\Gamma$ :

$$
v_{n}(s)=\frac{e^{i n s / L}}{\sqrt{2 \pi L}} \quad(n \in \mathbb{Z}),
$$

where the curvilinear coordinate $s$ is related to the polar angle $\phi$ as $s=\phi L$. The associated eigenvalues are

$$
\mu_{n}^{(p)}=g_{n}^{\prime}(L) \quad(n \in \mathbb{Z}) .
$$

The eigenfunctions do not depend on $p$, whereas the eigenvalues are twice degenerate, except for $n=0$. Here, the index $n$ runs over all integer numbers. In the limit $p \rightarrow 0$, one gets

$$
\mu_{n}^{(0)}=\frac{|n|}{L} \frac{1-(R / L)^{2|n|}}{1+(R / L)^{2|n|}} .
$$

The spectral decomposition (12) of the surface hopping propagator reads

$$
\Sigma_{p}\left(s, \ell \mid s_{0}\right)=\sum_{n=-\infty}^{\infty} \frac{e^{i n\left(s_{0}-s\right) / L}}{2 \pi L} \exp \left(-\mu_{n}^{(p)} \ell\right) .
$$

To access the full propagator, one also needs to compute $V_{n}^{(p)}\left(\boldsymbol{x}_{0}\right)$ from Eq. (19). Using the summation formulas from [69], the Laplace-transformed propagator $\tilde{G}_{\infty}\left(\boldsymbol{x}, p \mid \boldsymbol{x}_{0}\right)$ and thus $\tilde{j}_{\infty}\left(\boldsymbol{s}, p \mid \boldsymbol{x}_{0}\right)$ for a circular annulus with Dirichlet boundary condition on the outer circle and Neumann boundary condition on the inner circle read

$$
\begin{aligned}
\tilde{G}_{\infty}\left(\boldsymbol{x}, p \mid \boldsymbol{x}_{0}\right) & =\frac{1}{2 \pi D} \sum_{n=-\infty}^{\infty} e^{i n\left(\phi-\phi_{0}\right)} g_{n}\left(r_{0}\right) \\
& \times\left[K_{n}(\alpha L) I_{n}(\alpha r)-I_{n}(\alpha L) K_{n}(\alpha r)\right] \\
\tilde{j}_{\infty}\left(s, p \mid \boldsymbol{x}_{0}\right) & =\frac{1}{2 \pi L} \sum_{n=-\infty}^{\infty} e^{i n\left(\phi-\phi_{0}\right)} g_{n}\left(r_{0}\right)
\end{aligned}
$$

where $\boldsymbol{x}=(r, \phi)$ and $\boldsymbol{x}_{0}=\left(r_{0}, \phi_{0}\right)$ in polar coordinates, $R \leq r_{0} \leq r \leq L, s=\phi L$, and we used the Wronskian

$$
I_{n}^{\prime}(z) K_{n}(z)-K_{n}^{\prime}(z) I_{n}(z)=\frac{1}{z} .
$$

The projection of $\tilde{j}_{\infty}\left(s, p \mid \boldsymbol{x}_{0}\right)$ onto an eigenfunction $v_{n}(s)$ from Eq. (A3) reads then

$$
V_{n}^{(p)}\left(\boldsymbol{x}_{0}\right)=v_{n}\left(\phi_{0}\right) g_{n}\left(r_{0}\right) .
$$

The orthogonality of Fourier harmonics to a constant function reduces Eq. (23) to

$$
\tilde{U}\left(\ell, p \mid \boldsymbol{x}_{0}\right)=g_{0}\left(r_{0}\right) \exp \left(-\mu_{0}^{(p)} \ell\right),
$$

from which Eq. (22) gives the Laplace-transformed probability density of the reaction time as

$$
\tilde{H}_{q}\left(p \mid \boldsymbol{x}_{0}\right)=g_{0}\left(r_{0}\right) \frac{1}{1+\mu_{0}^{(p)} / q} .
$$

\section{Interior of a disk}

In the limit $R \rightarrow 0$, the inner boundary shrinks to a point, and one gets the solution for the interior of a disk of radius $L: \Omega=\left\{\boldsymbol{x} \in \mathbb{R}^{2}:|\boldsymbol{x}|<L\right\}$ (see also [68]). The radial functions become

$$
g_{n}(r)=\frac{I_{n}(r \sqrt{p / D})}{I_{n}(L \sqrt{p / D})},
$$

while the eigenvalues and eigenfunctions of the Dirichletto-Neumann operator are still given by Eqs. (A3, A4). Other expressions are also valid; in particular, Eqs. (A7, A8) are applicable.

$$
\begin{aligned}
& \text { At } p=0, \text { Eq. (A5) yields } \mu_{n}^{(0)}=|n| / L \text { and thus } \\
& \Sigma_{0}\left(s, \ell \mid s_{0}\right)=\frac{1-e^{-2 \ell / L}}{2 \pi L\left(1-2 \cos \left(\frac{s-s_{0}}{L}\right) e^{-\ell / L}+e^{-2 \ell / L}\right)} .
\end{aligned}
$$

As expected, this propagator evolves from the Dirac distribution $\delta\left(s-s_{0}\right)$ at $\ell=0$ to the uniform distribution $1 /(2 \pi L)$ as $\ell \rightarrow \infty$. Setting $\rho=L e^{-\ell / L}$, one can recognize in this form the Poisson kernel in the disk of radius $L$. The Poisson kernel describes the harmonic measure density on the disk, i.e., the probability density of the first arrival onto the circle of radius $R$ at point $(L, s / L)$ for Brownian motion started from a point $\left(\rho, s_{0} / L\right)$ (written in polar coordinates). As in the planar case discussed in Sec. III A, the distribution of the position of the diffusing particle at the boundary local time $\ell$ (i.e., after a prescribed number of encounters with the reflecting circle) is identical to the distribution of the first arrival point on the fully absorbing circle, where the boundary local time $\ell$ determines the starting point in the latter setting. Figure 3(a,b) illustrates the behavior of the surface hopping propagator.

\section{Reactive inner circle}

We briefly discuss the case (iii) when the inner circle is reactive and surrounded by a reflecting outer circle. This 
is a typical setting of a small reactive target confined in a domain surrounded by an outer reflecting boundary [70]. Here, we search for the spectrum of the Dirichlet-toNeumann operator associated with the inner circle: $\Gamma=$ $\left\{\boldsymbol{x} \in \mathbb{R}^{2}:|\boldsymbol{x}|=R\right\}$. Repeating the above construction step by step, one realizes that the eigenfunctions are the Fourier harmonics on the inner circle

$$
v_{n}(s)=\frac{e^{i n s / R}}{\sqrt{2 \pi R}} \quad(n \in \mathbb{Z}),
$$

where the curvilinear coordinate $s$ is related to the polar angle $\phi$ as $s=\phi R$. The associated eigenvalues are

$$
\mu_{n}^{(p)}=-g_{n}^{\prime}(R) \quad(n \in \mathbb{Z}),
$$

where sign minus appears due to the direction of the normal derivative, $\partial_{\boldsymbol{n}}=-\partial_{r}$, and

$$
g_{n}(r)=\frac{K_{n}^{\prime}(\alpha L) I_{n}(\alpha r)-I_{n}^{\prime}(\alpha L) K_{n}(\alpha r)}{K_{n}^{\prime}(\alpha L) I_{n}(\alpha R)-I_{n}^{\prime}(\alpha L) K_{n}(\alpha R)}
$$

are the radial functions satisfying $g_{n}(R)=1$ and $g_{n}^{\prime}(L)=$ 0 . In the limit $p \rightarrow 0$, one gets

$$
\mu_{n}^{(0)}=\frac{|n|}{R} \frac{1-(R / L)^{2|n|}}{1+(R / L)^{2|n|}} .
$$

The spectral decomposition (12) of the surface hopping propagator reads

$$
\Sigma_{p}\left(s, \ell \mid s_{0}\right)=\sum_{n=-\infty}^{\infty} \frac{e^{i n\left(s_{0}-s\right) / R}}{2 \pi R} \exp \left(-\mu_{n}^{(p)} \ell\right) .
$$

The Laplace-transformed propagator $\tilde{G}_{\infty}\left(\boldsymbol{x}, p \mid \boldsymbol{x}_{0}\right)$ and thus $\tilde{j}_{\infty}\left(\boldsymbol{s}, p \mid \boldsymbol{x}_{0}\right)$ for a circular annulus with Dirichlet boundary condition on the inner circle and Neumann boundary condition on the outer circle read

$$
\begin{aligned}
\tilde{G}_{\infty}\left(\boldsymbol{x}, p \mid \boldsymbol{x}_{0}\right) & =\frac{1}{2 \pi D} \sum_{n=-\infty}^{\infty} e^{i n\left(\phi-\phi_{0}\right)} g_{n}\left(r_{0}\right) \\
& \times\left[K_{n}(\alpha R) I_{n}(\alpha r)-I_{n}(\alpha R) K_{n}(\alpha r)\right] \\
\tilde{j}_{\infty}\left(s, p \mid \boldsymbol{x}_{0}\right) & =\frac{1}{2 \pi R} \sum_{n=-\infty}^{\infty} e^{i n\left(\phi-\phi_{0}\right)} g_{n}\left(r_{0}\right)
\end{aligned}
$$

where $\boldsymbol{x}=(r, \phi), \boldsymbol{x}_{0}=\left(r_{0}, \phi_{0}\right), R \leq r \leq r_{0} \leq L, s=\phi R$. In turn, Eqs. (A10, A11, A12) remain unchanged. Some first-passage properties in this setting were studied in [34].

\section{Exterior of a disk}

In the limit $L \rightarrow \infty$, the outer boundary is pushed away to infinity, and one deals with diffusion in the exterior of a disk of radius $R: \Omega=\left\{\boldsymbol{x} \in \mathbb{R}^{2}:|\boldsymbol{x}|>R\right\}$.
In this limit, the radial functions from Eq. (A17) are reduced to

$$
g_{n}(r)=\frac{K_{n}(r \sqrt{p / D})}{K_{n}(R \sqrt{p / D})}
$$

The eigenvalues and eigenfunctions of the Dirichlet-toNeumann operator are still given by Eqs. (A16, A15), with $g_{n}(r)$ from Eq. (A22). Other earlier expressions are as well applicable; in particular, Eqs. (A20, A21) are valid. Figure 3(c,d) illustrates the behavior of the surface hopping propagator.

The exterior of a disk presents a convenient example to illustrate subtle points of recurrent diffusion outside a planar bounded domain. As the radial functions $g_{n}(r)$ in Eq. (A22) for $p>0$ vanish exponentially fast in the limit $r \rightarrow \infty$, a general solution $w(r, \phi)$ of the modified Helmholtz equation (27) also vanishes, in agreement with the regularity condition. In the case $p=0$, the radial functions for $n \neq 0$ become $g_{n}(r)=(R / r)^{|n|}$ and vanish again. However, the limit of $g_{0}(r)$ as $p \rightarrow 0$ is equal to 1 that does not vanish at infinity, thus violating the regularity condition. This is a consequence of the simple fact that the rotationally invariant Laplace equation in the plane, $w^{\prime \prime}+\frac{1}{r} w^{\prime}=0$, has a general solution $c_{1}+c_{2} \ln r$ that does not vanish as $r \rightarrow \infty$, except for the trivial choice $c_{1}=c_{2}=0$. This is a well-known problem for such planar domains, for which, in particular, there is no steady-state reaction rate $[71,72]$. In the remaining part of the paper, we do not discuss this subtle case.

\section{Both reactive circles}

When both circles are reactive, one needs to consider the Dirichlet-to-Neumann operator on the whole boundary composed of two disjoint circles: $\Gamma_{1}=\{\boldsymbol{x} \in$ $\left.\mathbb{R}^{2}:|\boldsymbol{x}|=R\right\}$ and $\Gamma_{2}=\left\{\boldsymbol{x} \in \mathbb{R}^{2}:|\boldsymbol{x}|=L\right\}$. A general solution of Eq. (9) can be searched in the form

$$
w(r, \phi)=\sum_{n=-\infty}^{\infty}\left(c_{n, 1} g_{n, 1}(r)+c_{n, 2} g_{n, 2}(r)\right) e^{i n \phi},
$$

where the unknown coefficients $c_{n, 1}$ and $c_{n, 2}$ are set by boundary conditions, and the radial functions

$$
\begin{aligned}
g_{n, 1}(r) & =\frac{K_{n}(\alpha L) I_{n}(\alpha r)-I_{n}(\alpha L) K_{n}(\alpha r)}{K_{n}(\alpha L) I_{n}(\alpha R)-I_{n}(\alpha L) K_{n}(\alpha R)} \\
g_{n, 2}(r) & =\frac{K_{n}(\alpha R) I_{n}(\alpha r)-I_{n}(\alpha R) K_{n}(\alpha r)}{K_{n}(\alpha R) I_{n}(\alpha L)-I_{n}(\alpha R) K_{n}(\alpha L)}
\end{aligned}
$$

satisfy $g_{n, 1}(L)=0, g_{n, 1}(R)=1$, and $g_{n, 2}(R)=0$, $g_{n, 2}(L)=1$ for convenience (other linear combinations of $I_{n}(\alpha r)$ and $K_{n}(\alpha r)$ could also be used). Note that $g_{n, 1}(r)$ monotonously decreases, whereas $g_{n, 2}(r)$ monotonously increases on the interval $(R, L)$.

As the inner and outer circles are concentric, one can expect that an eigenfunction of the operator $\mathcal{M}_{p}$ can be 
written as

$$
v_{n}^{(p)}(s)= \begin{cases}a_{n}^{(p)} e^{i n \phi} & s \in \Gamma_{1} \quad(\phi=s / R), \\ b_{n}^{(p)} e^{i n \phi} & s \in \Gamma_{2} \quad(\phi=s / L),\end{cases}
$$

where $a_{n}^{(p)}$ and $b_{n}^{(p)}$ are some coefficients. In fact, as the space of functions on the whole boundary $\partial \Omega=\Gamma_{1} \cup \Gamma_{2}$ is the direct product of spaces of functions on the inner $\left(\Gamma_{1}\right)$ and outer $\left(\Gamma_{2}\right)$ circles, the eigenfunction $v_{n}^{(p)}(s)$ can be thought of being composed of two components. Here, these two components are proportional to $e^{i n \phi}$ due to the rotational symmetry (note that this claim can be shown rigorously by representing each component as a Fourier series and then using the orthogonality of Fourier harmonics). Substituting such $v_{n}^{(p)}(s)$ as the Dirichlet condition to Eq. (9), one gets its solution as

$$
w(r, \phi)=\left(a_{n}^{(p)} g_{n, 1}(r)+b_{n}^{(p)} g_{n, 2}(r)\right) e^{i n \phi},
$$

while its normal derivative on $\partial \Omega$ reads

$$
\begin{aligned}
\mathcal{M}_{p} v_{n} & =\left(\partial_{n} w\right)_{\mid \partial \Omega} \\
& = \begin{cases}-\left(a_{n}^{(p)} g_{n, 1}^{\prime}(R)+b_{n}^{(p)} g_{n, 2}^{\prime}(R)\right) e^{i n \phi} & s \in \Gamma_{1}, \\
\left(a_{n}^{(p)} g_{n, 1}^{\prime}(L)+b_{n}^{(p)} g_{n, 2}^{\prime}(L)\right) e^{i n \phi} & s \in \Gamma_{2} .\end{cases}
\end{aligned}
$$

If the right-hand side is proportional to $v_{n}^{(p)}$, then $v_{n}^{(p)}$ is indeed an eigenfunction of $\mathcal{M}_{p}$. In other words, we get two equations

$$
\left\{\begin{array}{l}
-\left(a_{n}^{(p)} g_{n, 1}^{\prime}(R)+b_{n}^{(p)} g_{n, 2}^{\prime}(R)\right)=\mu_{n}^{(p)} a_{n}^{(p)}, \\
\left(a_{n}^{(p)} g_{n, 1}^{\prime}(L)+b_{n}^{(p)} g_{n, 2}^{\prime}(L)\right)=\mu_{n}^{(p)} b_{n}^{(p)},
\end{array}\right.
$$

where the proportionality coefficient $\mu_{n}^{(p)}$ is the associated eigenvalue, and we used the particular form of functions $g_{n, 1}(r)$ and $g_{n, 2}(r)$. These equations can be written in a matrix form as

$$
\left(\begin{array}{cc}
-g_{n, 1}^{\prime}(R) & -g_{n, 2}^{\prime}(R) \\
g_{n, 1}^{\prime}(L) & g_{n, 2}^{\prime}(L)
\end{array}\right)\left(\begin{array}{c}
a_{n}^{(p)} \\
b_{n}^{(p)}
\end{array}\right)=\mu_{n}^{(p)}\left(\begin{array}{c}
a_{n}^{(p)} \\
b_{n}^{(p)}
\end{array}\right) .
$$

Solving this eigenvalue problem for the $2 \times 2$ matrix on the left-hand side, one determines the eigenvalue $\mu_{n}^{(p)}$, as well as one of the coefficients (e.g., $\left.a_{n}^{(p)}\right)$. The other coefficient (e.g., $b_{n}^{(p)}$ ) is fixed by imposing the $L_{2}(\partial \Omega)$ normalization of the eigenfunction $v_{n}^{(p)}$.

The Wronskian (A9) yields

$$
g_{n, 1}^{\prime}(L)=\frac{A}{L}, \quad g_{n, 2}^{\prime}(R)=-\frac{A}{R},
$$

where

$$
A=\frac{1}{K_{n}(\alpha L) I_{n}(\alpha R)-I_{n}(\alpha L) K_{n}(\alpha R)} .
$$

The eigenvalue $\mu_{n}^{(p)}$ is then obtained as a solution of the quadratic equation

$$
\mu^{2}+\mu\left(g_{n, 1}^{\prime}(R)-g_{n, 2}^{\prime}(L)\right)+B=0,
$$

where

$$
\begin{aligned}
B & =-g_{n, 1}^{\prime}(R) g_{n, 2}^{\prime}(L)+g_{n, 1}^{\prime}(L) g_{n, 2}^{\prime}(R) \\
& =-\alpha^{2} \frac{K_{n}^{\prime}(\alpha L) I_{n}^{\prime}(\alpha R)-I_{n}^{\prime}(\alpha L) K_{n}^{\prime}(\alpha R)}{K_{n}(\alpha L) I_{n}(\alpha R)-I_{n}(\alpha L) K_{n}(\alpha R)} .
\end{aligned}
$$

With the help of Eq. (A29), it is easy to check that the determinant of this equation is positive, so that there are two real roots. Moreover, as $B>0$, both roots are positive:

$$
\begin{aligned}
\mu_{n, \pm}^{(p)} & =\frac{1}{2}\left(g_{n, 2}^{\prime}(L)-g_{n, 1}^{\prime}(R)\right. \\
& \left. \pm \sqrt{\left(g_{n, 1}^{\prime}(R)+g_{n, 2}^{\prime}(L)\right)^{2}-4 g_{n, 1}^{\prime}(L) g_{n, 2}^{\prime}(R)}\right) .
\end{aligned}
$$

As a consequence, for each index $n$, there are two distinct eigenmodes. For each of them, the coefficients are determined by the corresponding eigenvalue. To avoid round-off errors in practical implementation, it is convenient to use slightly different (but formally equivalent) representation for + and - modes. In fact, we set

$$
a_{n,+}^{(p)}=-c_{n,+}^{(p)}\left(g_{n, 2}^{\prime}(L)-\mu_{n,+}^{(p)}\right), \quad b_{n,+}^{(p)}=c_{n,+}^{(p)} g_{n, 1}^{\prime}(L),
$$

where $c_{n,+}^{(p)}$ is fixed by the normalization of the eigenfunction:

$$
\begin{aligned}
1 & =\int_{\partial \Omega} d s\left|v_{n,+}^{(p)}(s)\right|^{2} \\
& =\left[c_{n,+}^{(p)}\right]^{2}\left(2 \pi R\left[g_{n, 2}^{\prime}(L)-\mu_{n,+}^{(p)}\right]^{2}+2 \pi L\left[g_{n, 1}^{\prime}(L)\right]^{2}\right) .
\end{aligned}
$$

In turn, for the mode with $\mu_{n,-}^{(p)}$, one can use

$a_{n,-}^{(p)}=c_{n,-}^{(p)} g_{n, 2}^{\prime}(R), \quad b_{n,-}^{(p)}=-c_{n,-}^{(p)}\left(g_{n, 1}^{\prime}(R)+\mu_{n,-}^{(p)}\right)$,

with

$$
1=\left[c_{n,-}^{(p)}\right]^{2}\left(2 \pi R\left[g_{n, 2}^{\prime}(R)\right]^{2}+2 \pi L\left[g_{n, 1}^{\prime}(R)+\mu_{n,-}^{(p)}\right]^{2}\right) .
$$

In the limit $p \rightarrow 0$, one has

$$
\begin{aligned}
& g_{n, 1}^{\prime}(R) \rightarrow-\frac{|n|}{R} \frac{1+(R / L)^{2|n|}}{1-(R / L)^{2|n|}}, \\
& g_{n, 1}^{\prime}(L) \rightarrow-\frac{|n|}{L} \frac{2(R / L)^{|n|}}{1-(R / L)^{2|n|}}, \\
& g_{n, 2}^{\prime}(R) \rightarrow \frac{|n|}{R} \frac{2(R / L)^{|n|}}{1-(R / L)^{2|n|}}, \\
& g_{n, 2}^{\prime}(L) \rightarrow \frac{|n|}{L} \frac{1+(R / L)^{2|n|}}{1-(R / L)^{2|n|}},
\end{aligned}
$$

so that

$$
\begin{aligned}
\mu_{n, \pm}^{(0)} & =\frac{|n|}{2} \frac{1+\gamma_{n}}{1-\gamma_{n}}\left(\frac{1}{L}+\frac{1}{R}\right. \\
& \left. \pm \sqrt{\left(\frac{1}{L}+\frac{1}{R}\right)^{2}-\frac{4\left(1-\gamma_{n}\right)^{2}}{L R\left(1+\gamma_{n}\right)^{2}}}\right)
\end{aligned}
$$


where $\gamma_{n}=(R / L)^{2|n|}$. In the case $n=0$, one can take the limit $n \rightarrow 0$ to get

$$
\mu_{0,-}^{(0)}=0, \quad \mu_{0,+}^{(0)}=\frac{1 / L+1 / R}{\ln (L / R)} .
$$

We also get

$$
\begin{aligned}
& a_{0,-}^{(0)}=b_{0,-}^{(0)}=\frac{1}{\sqrt{2 \pi(R+L)}}, \\
& a_{0,+}^{(0)}=\frac{1}{\sqrt{2 \pi R(1+R / L)}}, \quad b_{0,+}^{(0)}=\frac{-1}{\sqrt{2 \pi L(1+L / R)}} .
\end{aligned}
$$

Using the asymptotic behavior of the modified Bessel functions, one can check that

$$
\begin{aligned}
& \lim _{R \rightarrow 0} \mu_{n,-}^{(p)}=\sqrt{p / D} \frac{I_{n}^{\prime}(L \sqrt{p / D})}{I_{n}(L \sqrt{p / D})}, \\
& \lim _{R \rightarrow 0} \mu_{n,+}^{(p)}=+\infty \\
& \lim _{L \rightarrow \infty} \mu_{n,-}^{(p)}=-\sqrt{p / D} \frac{K_{n}^{\prime}(R \sqrt{p / D})}{K_{n}(R \sqrt{p / D})}, \\
& \lim _{L \rightarrow \infty} \mu_{n,+}^{(p)}=\sqrt{p / D}
\end{aligned}
$$

where we used that $K_{n}^{\prime}(z) / K_{n}(z) \leq-1$. In the limit $R \rightarrow 0, \mu_{n,-}^{(p)}$ approach the eigenvalues of $\mathcal{M}_{p}$ for the interior of a disk of radius $L$, whereas $\mu_{n,+}^{(p)}$ diverge and thus do not contribute. The opposite limit $L \rightarrow \infty$ is more subtle: $\mu_{n,+}^{(p)}$ approach the eigenvalues of $\mathcal{M}_{p}$ for the exterior of a disk of radius $R$; however, $\mu_{n,-}^{(p)}$ accumulate near $\sqrt{p / D}$.

Finally, the Dirichlet propagator in the Laplace domain is

$$
\begin{aligned}
& \tilde{G}_{\infty}\left(\boldsymbol{x}, p \mid \boldsymbol{x}_{0}\right)=\frac{1}{2 \pi D} \sum_{n=-\infty}^{\infty} e^{i n\left(\phi-\phi_{0}\right)} g_{n, 2}\left(r_{0}\right) \\
& \times\left[K_{n}(\alpha L) I_{n}(\alpha r)-I_{n}(\alpha L) K_{n}(\alpha r)\right] \quad\left(r_{0} \leq r\right), \\
& =\frac{1}{2 \pi D} \sum_{n=-\infty}^{\infty} e^{i n\left(\phi-\phi_{0}\right)} g_{n, 1}\left(r_{0}\right) \\
& \times\left[K_{n}(\alpha R) I_{n}(\alpha r)-I_{n}(\alpha R) K_{n}(\alpha r)\right] \quad\left(r \leq r_{0}\right),
\end{aligned}
$$

where $\boldsymbol{x}=(r, \phi)$ and $\boldsymbol{x}_{0}=\left(r_{0}, \phi_{0}\right)$. The probability flux density reads then

$$
\begin{aligned}
\tilde{j}_{\infty}\left(s, p \mid \boldsymbol{x}_{0}\right) & =\frac{1}{2 \pi R} \sum_{n=-\infty}^{\infty} e^{i n\left(\phi-\phi_{0}\right)} g_{n, 1}\left(r_{0}\right) \quad\left(s \in \Gamma_{1}\right), \\
& =\frac{1}{2 \pi L} \sum_{n=-\infty}^{\infty} e^{i n\left(\phi-\phi_{0}\right)} g_{n, 2}\left(r_{0}\right) \quad\left(s \in \Gamma_{2}\right) .
\end{aligned}
$$

As a consequence, one gets

$$
V_{n}^{(p)}\left(\boldsymbol{x}_{0}\right)=\left(a_{n}^{(p)} g_{n, 1}\left(r_{0}\right)+b_{n}^{(p)} g_{n, 2}\left(r_{0}\right)\right) e^{i n \phi_{0}}
$$

that gives access to the full propagator $P\left(\boldsymbol{x}, \ell, t \mid \boldsymbol{x}_{0}\right)$.

\section{Cylindrical domains}

The above analysis can also be extended to cylindrical domains. Let us first consider an infinite cylinder, $\Omega=\Omega_{0} \times \mathbb{R}$, where $\Omega_{0}$ is a disk of radius $L$. As the boundary $\partial \Omega$ is unbounded, the spectrum of the Dirichlet-toNeumann operator $\mathcal{M}_{p}$ is not discrete anymore. Nevertheless, the symmetries of this domain admit the separation of variables and allow for getting "eigenfunctions" and "eigenvalues" in cylindrical coordinates as

$$
\begin{array}{rlrl}
v_{n k}(\phi, z) & =\frac{e^{i n \phi+i k z}}{2 \pi \sqrt{L}} \quad & (n \in \mathbb{Z}, k \in \mathbb{R}), \\
\mu_{n k}^{(p)}=\alpha \frac{I_{n}^{\prime}(\alpha L)}{I_{n}(\alpha L)}, & \alpha=\sqrt{p / D+k^{2}} .
\end{array}
$$

The surface hopping propagator reads then

$$
\Sigma_{p}\left(\boldsymbol{s}, \ell \mid \boldsymbol{s}_{0}\right)=\sum_{n=-\infty}^{\infty} \frac{e^{i n\left(\phi_{0}-\phi\right)}}{2 \pi L} \int_{\mathbb{R}} \frac{d k}{2 \pi} e^{i k\left(z_{0}-z\right)} e^{-\mu_{n k}^{(p)} \ell}
$$

where $\boldsymbol{s}=(L, \phi, z)$ and $\boldsymbol{s}_{0}=\left(L, \phi_{0}, z_{0}\right)$ in cylindrical coordinates. If $\Omega_{0}$ is a circular annulus, one has to use the appropriate radial function $g_{n}(r)$, with $\alpha=\sqrt{p / D+k^{2}}$. Other related quantities can also be obtained.

When the cylinder is finite, $\Omega=\Omega_{0} \times(0, b)$, the spectrum of $\mathcal{M}_{p}$ is discrete again, but the analysis is more subtle. In fact, as in the case of a circular annulus, different combinations of reactivity patterns are possible: all the boundary is reactive; only the lateral boundary is reactive but the top and bottom disks are reflecting; only the top disk is reactive but the remaining boundary is reflecting; etc. When only one part of the boundary is reactive, the analysis is rather simple. For instance, if only the lateral boundary is reactive, the eigenfunctions and eigenvalues are

$$
\begin{aligned}
v_{n k}(\phi, z) & =\frac{e^{i n \phi}}{\sqrt{2 \pi L}} \frac{\sqrt{2-\delta_{n, 0}}}{\sqrt{b}} \cos (\pi k z / b), \\
\mu_{n k}^{(p)} & =\alpha \frac{I_{n}^{\prime}(\alpha L)}{I_{n}(\alpha L)}, \quad \alpha=\sqrt{p / D+(\pi k / b)^{2}}
\end{aligned}
$$

with $n \in \mathbb{Z}$ and $k=0,1,2, \ldots$. However, the analysis is more involved when the whole boundary is reactive.

\section{Appendix B: Spherical shell}

In three dimensions, one can consider a spherical shell between two concentric spheres of radii $R<L$ : $\Omega=$ $\left\{\boldsymbol{x} \in \mathbb{R}^{3}: R<|\boldsymbol{x}|<L\right\}$, with three combinations of boundary conditions. As the analysis is rather similar to the two-dimensional setting, the results are presented in a concise form. 


\section{Reactive outer sphere}

As previously, we start with the case of the Dirichletto-Neumann operator $\mathcal{M}_{p}$ associated with the reactive outer sphere $\Gamma=\left\{\boldsymbol{x} \in \mathbb{R}^{3}:|\boldsymbol{x}|=L\right\}$. The rotational invariance implies that the eigenfunctions of $\mathcal{M}_{p}$ are the (normalized) spherical harmonics,

$$
v_{n m}(\boldsymbol{s})=\frac{1}{L} Y_{m n}(\theta, \phi) \quad(n=0,1,2, \ldots,|m| \leq n) .
$$

The eigenvalues are obtained by solving the mixed boundary value problem (27):

$$
\mu_{n}^{(p)}=g_{n}^{\prime}(R) \quad(n=0,1,2, \ldots),
$$

where

$$
g_{n}(r)=\frac{k_{n}^{\prime}(\alpha R) i_{n}(\alpha r)-i_{n}^{\prime}(\alpha R) k_{n}(\alpha r)}{k_{n}^{\prime}(\alpha R) i_{n}(\alpha L)-i_{n}^{\prime}(\alpha R) k_{n}(\alpha L)},
$$

$\alpha=\sqrt{p / D}$, and

$$
\begin{aligned}
& i_{n}(z)=\sqrt{\pi /(2 z)} I_{n+1 / 2}(z), \\
& k_{n}(z)=\sqrt{2 /(\pi z)} K_{n+1 / 2}(z)
\end{aligned}
$$

are the modified spherical Bessel functions of the first and second kind, respectively. The eigenfunctions do not depend on $p$, whereas the eigenvalues $\mu_{n}^{(p)}$ do not depend on the second index $m$ and are thus $(2 n+1)$ times degenerate. In the limit $p \rightarrow 0$, one gets

$$
\mu_{n}^{(0)}=\frac{n(n+1)}{L} \frac{1-(R / L)^{2 n+1}}{n+1+n(R / L)^{2 n+1}} .
$$

The surface hopping propagator from Eq. (12) reads

$\Sigma_{p}\left(\boldsymbol{s}, \ell \mid \boldsymbol{s}_{0}\right)=\frac{1}{L^{2}} \sum_{n=0}^{\infty} \sum_{m=-n}^{n} Y_{m n}^{*}\left(\theta_{0}, \phi_{0}\right) Y_{m n}(\theta, \phi) e^{-\mu_{n}^{(p)} \ell}$.

Since the eigenvalues do not depend on the index $m$, one can apply the addition theorem for spherical harmonics to evaluate the sum over $m$ :

$$
\Sigma_{p}\left(\boldsymbol{s}, \ell \mid \boldsymbol{s}_{0}\right)=\frac{1}{4 \pi L^{2}} \sum_{n=0}^{\infty}(2 n+1) P_{n}\left(\frac{\left(\boldsymbol{s} \cdot \boldsymbol{s}_{0}\right)}{|\boldsymbol{s}|\left|\boldsymbol{s}_{0}\right|}\right) e^{-\mu_{n}^{(p)} \ell},
$$

where $P_{n}(z)$ are Legendre polynomials.

One also needs to compute $V_{n}^{(p)}\left(\boldsymbol{x}_{0}\right)$ from Eq. (19). Using the summation formulas from [69], the Laplacetransformed quantities $\tilde{G}_{\infty}\left(\boldsymbol{x}, p \mid \boldsymbol{x}_{0}\right)$ and thus $\tilde{j}_{\infty}\left(\boldsymbol{s}, p \mid \boldsymbol{x}_{0}\right)$ for a spherical shell with Dirichlet boundary condition on the outer sphere and Neumann boundary condition on the inner sphere read

$$
\begin{aligned}
\tilde{G}_{\infty}\left(\boldsymbol{x}, p \mid \boldsymbol{x}_{0}\right) & =\sum_{n=0}^{\infty} \frac{\alpha(2 n+1)}{4 \pi D} P_{n}\left(\frac{\left(\boldsymbol{x} \cdot \boldsymbol{x}_{0}\right)}{|\boldsymbol{x}|\left|\boldsymbol{x}_{0}\right|}\right) g_{n}\left(r_{0}\right) \\
& \times\left[k_{n}(\alpha L) i_{n}(\alpha r)-i_{n}(\alpha L) k_{n}(\alpha r)\right], \\
\tilde{j}_{\infty}\left(\boldsymbol{s}, p \mid \boldsymbol{x}_{0}\right) & =\sum_{n=0}^{\infty} \frac{2 n+1}{4 \pi L^{2}} P_{n}\left(\frac{\left(\boldsymbol{s} \cdot \boldsymbol{x}_{0}\right)}{|\boldsymbol{s}|\left|\boldsymbol{x}_{0}\right|}\right) g_{n}\left(r_{0}\right),
\end{aligned}
$$

where $r=|\boldsymbol{x}|, r_{0}=\left|\boldsymbol{x}_{0}\right|, R \leq r_{0} \leq r \leq L$, and we used the Wronskian

$$
i_{n}^{\prime}(z) k_{n}(z)-k_{n}^{\prime}(z) i_{n}(z)=\frac{1}{z^{2}} .
$$

The projection of $\tilde{j}_{\infty}\left(\boldsymbol{s}, p \mid \boldsymbol{x}_{0}\right)$ onto an eigenfunction $v_{n m}(s)$ from Eq. (B1) reads then

$$
V_{n m}^{(p)}\left(\boldsymbol{x}_{0}\right)=v_{m n}\left(\theta_{0}, \phi_{0}\right) g_{n}\left(r_{0}\right)
$$

where $\boldsymbol{x}_{0}=\left(r_{0}, \theta_{0}, \phi_{0}\right)$ in spherical coordinates. The orthogonality of spherical harmonics reduces Eq. (23) to

$$
\tilde{U}\left(\ell, p \mid \boldsymbol{x}_{0}\right)=g_{0}\left(r_{0}\right) \exp \left(-\mu_{0}^{(p)} \ell\right)
$$

while the probability density of the reaction time reads

$$
\tilde{H}_{q}\left(p \mid \boldsymbol{x}_{0}\right)=g_{0}\left(r_{0}\right) \frac{1}{1+\mu_{0}^{(p)} / q} .
$$

\section{Interior of a ball}

In the limit $R \rightarrow 0$, the inner boundary shrinks to a point, and one gets the solution for the interior of a ball of radius $L: \Omega=\left\{\boldsymbol{x} \in \mathbb{R}^{3}:|\boldsymbol{x}|<L\right\}$, with radial functions

$$
g_{n}(r)=\frac{i_{n}(r \sqrt{p / D})}{i_{n}(L \sqrt{p / D})} .
$$

The eigenvalues and eigenfunctions of the Dirichlet-toNeumann operator are still given by Eqs. (B1, B2), and other earlier expressions remain valid; in particular, Eqs. $(\mathrm{B} 7, \mathrm{~B} 8)$ are applicable. At $p=0$, the eigenvalues are simply $\mu_{n}^{(0)}=n / L$, for which Eq. (B6) can be evaluated explicitly using the generating function of Legendre polynomials:

$$
\Sigma_{0}\left(\boldsymbol{s}, \ell \mid \boldsymbol{s}_{0}\right)=\frac{L}{4 \pi} \frac{1-e^{-2 \ell / L}}{\left|e^{-\ell / L} \boldsymbol{s}_{0}-\boldsymbol{s}\right|^{3}} .
$$

This expression coincides with the harmonic measure density on the sphere when the starting point is $\boldsymbol{s}_{0} e^{-\ell / L}$. It can also be written in terms of the angle $\theta$ between vectors $\boldsymbol{s}_{0}$ and $\boldsymbol{s}$ :

$$
\Sigma_{0}\left(\boldsymbol{s}, \ell \mid \boldsymbol{s}_{0}\right)=\frac{1}{4 \pi L^{2}} \frac{1-e^{-2 \ell / L}}{\left[1-2 e^{-\ell / L} \cos \theta+e^{-2 \ell / L}\right]^{3 / 2}} .
$$

Figure 5(a,b) illustrates the behavior of the surface hopping propagator.

The orthogonality of spherical harmonics reduces Eq. (21) to

$$
\tilde{U}\left(\ell, p \mid \boldsymbol{x}_{0}\right)=\frac{R}{r_{0}} \frac{\sinh \left(r_{0} \sqrt{p / D}\right)}{\sinh (R \sqrt{p / D})} \exp \left(-\mu_{0}^{(p)} \ell\right),
$$


with $\mu_{0}^{(p)}=\sqrt{p / D} \operatorname{ctanh}(R \sqrt{p / D})-1 / R$. Similarly, one has

$$
\tilde{H}_{q}\left(p \mid \boldsymbol{x}_{0}\right)=\frac{R}{r_{0}} \frac{\sinh \left(r_{0} \sqrt{p / D}\right)}{\sinh (R \sqrt{p / D})} \frac{1}{1+\mu_{0}^{(p)} / q},
$$

from which the inverse Laplace transform yields the standard spectral expansion for $H_{q}\left(t \mid \boldsymbol{x}_{0}\right)$.

\section{Reactive inner sphere}

The analysis for the reactive inner sphere is very similar. The eigenfunctions of the Dirichlet-to-Neumann operator associated with the inner sphere $\Gamma=\{\boldsymbol{x} \in$ $\left.\mathbb{R}^{3}:|\boldsymbol{x}|=R\right\}$ are again the spherical harmonics but with the prefactor $1 / R$ for a proper normalization:

$$
v_{n m}(s)=\frac{1}{R} Y_{m n}(\theta, \phi) \quad(n=0,1,2, \ldots,|m| \leq n) .
$$

The eigenvalues are

$$
\mu_{n}^{(p)}=-g_{n}^{\prime}(R) \quad(n=0,1,2, \ldots),
$$

where

$$
g_{n}(r)=\frac{k_{n}^{\prime}(\alpha L) i_{n}(\alpha r)-i_{n}^{\prime}(\alpha L) k_{n}(\alpha r)}{k_{n}^{\prime}(\alpha L) i_{n}(\alpha R)-i_{n}^{\prime}(\alpha L) k_{n}(\alpha R)} .
$$

In the limit $p \rightarrow 0$, one gets

$$
\mu_{n}^{(0)}=\frac{n(n+1)}{R} \frac{1-(R / L)^{2 n+1}}{n+(n+1)(R / L)^{2 n+1}} .
$$

The expression for the surface hopping propagator is almost identical to Eq. (B6):

$$
\Sigma_{p}\left(\boldsymbol{s}, \ell \mid \boldsymbol{s}_{0}\right)=\frac{1}{4 \pi R^{2}} \sum_{n=0}^{\infty}(2 n+1) P_{n}\left(\frac{\left(\boldsymbol{s} \cdot \boldsymbol{s}_{0}\right)}{|\boldsymbol{s}|\left|\boldsymbol{s}_{0}\right|}\right) e^{-\mu_{n}^{(p)} \ell} .
$$

The Laplace-transformed propagator $\tilde{G}_{\infty}\left(\boldsymbol{x}, p \mid \boldsymbol{x}_{0}\right)$ and thus $\tilde{j}_{\infty}\left(\boldsymbol{s}, p \mid \boldsymbol{x}_{0}\right)$ for a spherical shell with Dirichlet boundary condition on the inner sphere and Neumann boundary condition on the outer sphere read

$$
\begin{aligned}
\tilde{G}_{\infty}\left(\boldsymbol{x}, p \mid \boldsymbol{x}_{0}\right) & =\sum_{n=0}^{\infty} \frac{\alpha(2 n+1)}{4 \pi D} P_{n}\left(\frac{\left(\boldsymbol{x} \cdot \boldsymbol{x}_{0}\right)}{|\boldsymbol{x}|\left|\boldsymbol{x}_{0}\right|}\right) g_{n}\left(r_{0}\right) \\
& \times\left[k_{n}(\alpha R) i_{n}(\alpha r)-i_{n}(\alpha R) k_{n}(\alpha r)\right], \\
\tilde{j}_{\infty}\left(\boldsymbol{s}, p \mid \boldsymbol{x}_{0}\right) & =\sum_{n=0}^{\infty} \frac{2 n+1}{4 \pi R^{2}} P_{n}\left(\frac{\left(\boldsymbol{s} \cdot \boldsymbol{x}_{0}\right)}{|\boldsymbol{s}|\left|\boldsymbol{x}_{0}\right|}\right) g_{n}\left(r_{0}\right),
\end{aligned}
$$

where $r=|\boldsymbol{x}|, r_{0}=\left|\boldsymbol{x}_{0}\right|, R \leq r \leq r_{0} \leq L$, from which

$$
V_{n m}^{(p)}\left(\boldsymbol{x}_{0}\right)=v_{m n}\left(\theta_{0}, \phi_{0}\right) g_{n}\left(r_{0}\right) .
$$

These quantities determine the full propagator $P\left(\boldsymbol{x}, \ell, t \mid \boldsymbol{x}_{0}\right)$.

\section{Exterior of a ball}

In the limit $L \rightarrow \infty$, the outer boundary is pushed away to infinity, and one deals with diffusion in the exterior of a ball of radius $R: \Omega=\left\{\boldsymbol{x} \in \mathbb{R}^{3}:|\boldsymbol{x}|>R\right\}$. The radial functions are reduced to

$$
g_{n}(r)=\frac{k_{n}(r \sqrt{p / D})}{k_{n}(R \sqrt{p / D})},
$$

while the eigenvalues and eigenfunctions of the Dirichletto-Neumann operator are still given by Eqs. (B18, B19). Interestingly, the eigenvalues are just polynomials of $\sqrt{p / D}$, e.g., $\mu_{0}^{(p)}=(1+R \sqrt{p / D}) / R$. The above expressions are as well applicable; in particular, Eqs. (B23, B24) are valid.

At $p=0$, the eigenvalues in Eq. (B21) are simplified as $\mu_{n}^{(0)}=(n+1) / R$, and the surface hopping propagator can be computed explicitly as

$$
\Sigma_{0}\left(\boldsymbol{s}, \ell \mid \boldsymbol{s}_{0}\right)=\frac{R e^{-\ell / R}}{4 \pi} \frac{1-e^{-2 \ell / R}}{\left|e^{-\ell / R} \boldsymbol{s}_{0}-\boldsymbol{s}\right|^{3}} .
$$

If $L$ is replaced by $R$, this expression coincides with Eq. (B14), except for an extra factor $e^{-\ell / R}$ that accounts for the possibility of escaping to infinity. Figure 5(c,d) illustrates the behavior of the surface hopping propagator.

The orthogonality of spherical harmonics reduces Eq. (23) to

$$
\tilde{U}\left(\ell, p \mid \boldsymbol{x}_{0}\right)=\frac{R}{r_{0}} \exp \left(-\left(r_{0}-R+\ell\right) \sqrt{p / D}-\ell / R\right),
$$

from which the inverse Laplace transform yields

$$
U\left(\ell, t \mid \boldsymbol{x}_{0}\right)=\frac{R e^{-\ell / R}}{r_{0}} \frac{r_{0}-R+\ell}{\sqrt{4 \pi D t^{3}}} e^{-\left(r_{0}-R+\ell\right)^{2} /(4 D t)} .
$$

This is a rare example when the probability density $U\left(\ell, t \mid \boldsymbol{x}_{0}\right)$ is found in a simple closed form. Setting $\ell=0$, one retrieves the probability density of the first-passage time for a perfectly absorbing sphere [73]. In turn, the integral (22) yields the probability density of the firstpassage time to a partially reactive sphere $[12,70]$

$$
\begin{aligned}
H_{q}\left(t \mid \boldsymbol{x}_{0}\right) & =\frac{q D}{r_{0}} e^{-\left(r_{0}-R\right)^{2} /(4 D t)}\left\{\frac{R}{\sqrt{\pi D t}}\right. \\
& \left.-(1+q R) \operatorname{erfcx}\left(\frac{r_{0}-R}{\sqrt{4 D t}}+(1+q R) \frac{\sqrt{D t}}{R}\right)\right\} .
\end{aligned}
$$

\section{Both reactive spheres}

Finally, the analysis for both reactive spheres is the most involved but very similar to the planar case. For this reason, we just reproduce the main formulas adapted to the three-dimensional case. Here, one employs two 
families of radial functions,

$$
\begin{aligned}
g_{n, 1}(r) & =\frac{k_{n}(\alpha L) i_{n}(\alpha r)-i_{n}(\alpha L) k_{n}(\alpha r)}{k_{n}(\alpha L) i_{n}(\alpha R)-i_{n}(\alpha L) k_{n}(\alpha R)}, \\
g_{n, 2}(r) & =\frac{k_{n}(\alpha R) i_{n}(\alpha r)-i_{n}(\alpha R) k_{n}(\alpha r)}{k_{n}(\alpha R) i_{n}(\alpha L)-i_{n}(\alpha R) k_{n}(\alpha L)},
\end{aligned}
$$

which satisfy $g_{n, 1}(R)=1, g_{n, 1}(L)=0$ and $g_{n, 2}(L)=1$, $g_{n, 2}(R)=0$. The eigenfunctions are searched in the form

$$
v_{n m}^{(p)}(s)= \begin{cases}a_{n}^{(p)} Y_{m n}(\theta, \phi) & s \in \Gamma_{1}, \\ b_{n}^{(p)} Y_{m n}(\theta, \phi) & s \in \Gamma_{2},\end{cases}
$$

where $\Gamma_{1}$ and $\Gamma_{2}$ are the inner and the outer spheres forming the boundary $\partial \Omega$. The Wronskian (B9) implies

$$
g_{n, 1}^{\prime}(L)=\frac{A}{\alpha L^{2}}, \quad g_{n, 2}^{\prime}(R)=-\frac{A}{\alpha R^{2}},
$$

where

$$
A=\frac{1}{k_{n}(\alpha L) i_{n}(\alpha R)-i_{n}(\alpha L) k_{n}(\alpha R)} .
$$

In this case, one also has

$$
\begin{aligned}
B & =-g_{n, 1}^{\prime}(R) g_{n, 2}^{\prime}(L)+g_{n, 1}^{\prime}(L) g_{n, 2}^{\prime}(R) \\
& =-\alpha^{2} \frac{k_{n}^{\prime}(\alpha L) i_{n}^{\prime}(\alpha R)-i_{n}^{\prime}(\alpha L) k_{n}^{\prime}(\alpha R)}{k_{n}(\alpha L) i_{n}(\alpha R)-i_{n}(\alpha L) k_{n}(\alpha R)} .
\end{aligned}
$$

Using these expressions, one deduces again Eqs. (A33, A34) for the eigenvalue $\mu_{n, \pm}^{(p)}$ and the coefficients $a_{n, \pm}^{(p)}$ and $b_{n, \pm}^{(p)}$. The normalization coefficient $c_{n, \pm}^{(p)}$ is fixed by normalization:

$$
\begin{aligned}
& 1=\left[c_{n,+}^{(p)}\right]^{2}\left(4 \pi R^{2}\left[g_{n, 2}^{\prime}(L)-\mu_{n,+}^{(p)}\right]^{2}+4 \pi L^{2}\left[g_{n, 1}^{\prime}(L)\right]^{2}\right), \\
& 1=\left[c_{n,-}^{(p)}\right]^{2}\left(4 \pi R^{2}\left[g_{n, 2}^{\prime}(R)\right]^{2}+4 \pi L^{2}\left[g_{n, 1}^{\prime}(R)+\mu_{n,-}^{(p)}\right]^{2}\right) .
\end{aligned}
$$

In the limit $p \rightarrow 0$, one gets

$$
\begin{aligned}
& g_{n, 1}^{\prime}(R) \rightarrow-\frac{n+1+n(R / L)^{2 n+1}}{R\left(1-(R / L)^{2 n+1}\right)}, \\
& g_{n, 1}^{\prime}(L) \rightarrow-\frac{(2 n+1)(R / L)^{n+1}}{L\left(1-(R / L)^{2 n+1}\right)}, \\
& g_{n, 2}^{\prime}(R) \rightarrow \frac{(2 n+1)(R / L)^{n}}{R\left(1-(R / L)^{2 n+1}\right)}, \\
& g_{n, 2}^{\prime}(L) \rightarrow \frac{n+(n+1)(R / L)^{2 n+1}}{L\left(1-(R / L)^{2 n+1}\right)},
\end{aligned}
$$

(and $B \rightarrow n(n+1) /(L R))$, from which

$\mu_{n, \pm}^{(0)}=\frac{1}{2 R\left(1-\gamma_{n}\right)}\left(n(\beta+1)+1+\gamma_{n}(n+(n+1) \beta)\right.$

$\left.\pm \sqrt{\left(n(\beta+1)+1+\gamma_{n}(n+(n+1) \beta)\right)^{2}-4 \beta n(n+1)}\right)$, where $\beta=R / L$ and $\gamma_{n}=(R / L)^{2 n+1}$. We also get

$$
\mu_{0,-}^{(0)}=0, \quad \mu_{0,+}^{(0)}=\frac{1+\beta^{2}}{R(1-\beta)}
$$

and

$$
\begin{aligned}
& a_{0,-}^{(0)}=b_{0,-}^{(0)}=\frac{1}{\sqrt{4 \pi\left(R^{2}+L^{2}\right)}}, \\
& a_{0,+}^{(0)}=\frac{1}{R \sqrt{4 \pi\left(1+\beta^{2}\right)}}, \quad b_{0,+}^{(0)}=\frac{-\beta^{2}}{R \sqrt{4 \pi\left(1+\beta^{2}\right)}} .
\end{aligned}
$$

In the limit $L \rightarrow \infty$, one retrieves $\mu_{n,+}^{(0)} \rightarrow(n+1) / R$ and $\mu_{n,-}^{(0)} \rightarrow 0$. In turn, as $R \rightarrow 0$, one has $\mu_{n,-}^{(0)} \rightarrow n / L$, whereas $\mu_{n,+}^{(0)} \rightarrow \infty$.

Using the asymptotic behavior of the modified spherical Bessel functions, one can also check that

$$
\begin{aligned}
& \lim _{R \rightarrow 0} \mu_{n,-}^{(p)}=\sqrt{p / D} \frac{i_{n}^{\prime}(L \sqrt{p / D})}{i_{n}(L \sqrt{p / D})}, \\
& \lim _{R \rightarrow 0} \mu_{n,+}^{(p)}=\infty, \\
& \lim _{L \rightarrow \infty} \mu_{n,+}^{(p)}=-\sqrt{p / D} \frac{k_{n}^{\prime}(R \sqrt{p / D})}{k_{n}(R \sqrt{p / D})}, \\
& \lim _{L \rightarrow \infty} \mu_{n,-}^{(p)}=\sqrt{p / D},
\end{aligned}
$$

where we used that $k_{n}^{\prime}(z) / k_{n}(z) \leq-1$. As a consequence, in the limit $R \rightarrow 0$, one retrieves the eigenvalues of $\mathcal{M}_{p}$ for the interior of a ball of radius $L$. In turn, in the limit $L \rightarrow \infty, \mu_{n,+}^{(p)}$ approach the eigenvalues for the exterior of a ball of radius $R$, while $\mu_{n,-}^{(p)}$ accumulate near $\sqrt{p / D}$.

Finally, the Dirichlet propagator in the Laplace domain is

$$
\begin{gathered}
\tilde{G}_{\infty}\left(\boldsymbol{x}, p \mid \boldsymbol{x}_{0}\right)=\sum_{n=0}^{\infty} \frac{\alpha(2 n+1)}{4 \pi D} P_{n}\left(\frac{\left(\boldsymbol{x} \cdot \boldsymbol{x}_{0}\right)}{|\boldsymbol{x}|\left|\boldsymbol{x}_{0}\right|}\right) g_{n, 2}\left(r_{0}\right) \\
\times\left[k_{n}(\alpha L) i_{n}(\alpha r)-i_{n}(\alpha L) k_{n}(\alpha r)\right] \quad\left(r_{0} \leq r\right), \quad(\mathrm{B} 37 \\
=\sum_{n=0}^{\infty} \frac{\alpha(2 n+1)}{4 \pi D} P_{n}\left(\frac{\left(\boldsymbol{x} \cdot \boldsymbol{x}_{0}\right)}{|\boldsymbol{x}|\left|\boldsymbol{x}_{0}\right|}\right) g_{n, 1}\left(r_{0}\right) \\
\times\left[k_{n}(\alpha R) i_{n}(\alpha r)-i_{n}(\alpha R) k_{n}(\alpha r)\right] \quad\left(r \leq r_{0}\right),
\end{gathered}
$$

where $\boldsymbol{x}=(r, \theta, \phi)$ and $\boldsymbol{x}_{0}=\left(r_{0}, \theta_{0}, \phi_{0}\right)$. The probability flux density reads then

$$
\begin{aligned}
& \tilde{j}_{\infty}\left(\boldsymbol{s}, p \mid \boldsymbol{x}_{0}\right) \\
& =\sum_{n=0}^{\infty} \frac{2 n+1}{4 \pi R^{2}} P_{n}\left(\frac{\left(\boldsymbol{x} \cdot \boldsymbol{x}_{0}\right)}{|\boldsymbol{x}|\left|\boldsymbol{x}_{0}\right|}\right) g_{n, 1}\left(r_{0}\right) \quad\left(s \in \Gamma_{1}\right), \\
& =\sum_{n=0}^{\infty} \frac{2 n+1}{4 \pi R^{2}} P_{n}\left(\frac{\left(\boldsymbol{x} \cdot \boldsymbol{x}_{0}\right)}{|\boldsymbol{x}|\left|\boldsymbol{x}_{0}\right|}\right) g_{n, 2}\left(r_{0}\right) \quad\left(s \in \Gamma_{2}\right) .
\end{aligned}
$$


As a consequence, one gets

$$
V_{n}^{(p)}\left(\boldsymbol{x}_{0}\right)=\left(a_{n}^{(p)} g_{n, 1}\left(r_{0}\right)+b_{n}^{(p)} g_{n, 2}\left(r_{0}\right)\right) Y_{m n}\left(\theta_{0}, \phi_{0}\right)
$$

that gives access to the full propagator $P\left(\boldsymbol{x}, \ell, t \mid \boldsymbol{x}_{0}\right)$.
[1] S. Rice, Diffusion-Limited Reactions (Elsevier, Amsterdam, 1985).

[2] S. Redner, A Guide to First Passage Processes (Cambridge: Cambridge University press, 2001).

[3] Z. Schuss, Brownian Dynamics at Boundaries and Interfaces in Physics, Chemistry and Biology (Springer, New York, 2013).

[4] R. Metzler, G. Oshanin, and S. Redner (Eds.) FirstPassage Phenomena and Their Applications (Singapore: World Scientific, 2014).

[5] G. Oshanin, R. Metzler, K. Lindenberg (Eds.) Chemical Kinetics: Beyond the Textbook (New Jersey: World Scientific, 2019).

[6] D. S. Grebenkov, "NMR Survey of Reflected Brownian Motion", Rev. Mod. Phys. 79, 1077-1137 (2007).

[7] O. Bénichou, C. Loverdo, M. Moreau, and R. Voituriez, "Intermittent search strategies", Rev. Mod. Phys. 83, 81-130 (2011).

[8] P. C. Bressloff and J. M. Newby, "Stochastic models of intracellular transport", Rev. Mod. Phys. 85, 135-196 (2013).

[9] O. Bénichou and R. Voituriez, "From first-passage times of random walks in confinement to geometry-controlled kinetics", Phys. Rep. 539, 225-284 (2014).

[10] C. W. Gardiner, Handbook of stochastic methods for physics, chemistry and the natural sciences (Springer: Berlin, 1985).

[11] H. Risken, The Fokker-Planck equation: methods of solution and applications, 3rd Ed. (Berlin: Springer, 1996).

[12] F. C. Collins and G. E. Kimball, "Diffusion-controlled reaction rates", J. Coll. Sci. 4, 425 (1949).

[13] D. A. Lauffenburger and J. Linderman, Receptors: Models for Binding, Trafficking, and Signaling (Oxford University Press, 1993).

[14] H. Sano and M. Tachiya, "Partially diffusion-controlled recombination", J. Chem. Phys. 71, 1276-1282 (1979).

[15] H. Sano and M. Tachiya, "Theory of diffusion-controlled reactions on spherical surfaces and its application to reactions on micellar surfaces", J. Chem. Phys. 75, 2870-2878 (1981).

[16] D. Shoup and A. Szabo, "Role of diffusion in ligand binding to macromolecules and cell-bound receptors", Biophys. J. 40, 33-39 (1982).

[17] R. Zwanzig, "Diffusion-controlled ligand binding to spheres partially covered by receptors: an effective medium treatment", Proc. Natl. Acad. Sci. USA 87, 5856 (1990).

[18] B. Sapoval, "General Formulation of Laplacian Transfer Across Irregular Surfaces", Phys. Rev. Lett. 73, 33143317 (1994).

[19] M. Filoche and B. Sapoval, "Can One Hear the Shape of an Electrode? II. Theoretical Study of the Laplacian Transfer", Eur. Phys. J. B 9, 755-763 (1999).

[20] B. Sapoval, M. Filoche, and E. Weibel, "Smaller is better - but not too small: A physical scale for the design of the mammalian pulmonary acinus", Proc. Nat. Ac. Sci. USA 99, 10411-10416 (2002).

[21] D. S. Grebenkov, M. Filoche, and B. Sapoval, "Spectral Properties of the Brownian Self-Transport Operator", Eur. Phys. J. B 36, 221-231 (2003).

[22] A. Berezhkovskii, Y. Makhnovskii, M. Monine, V. Zitserman, and S. Shvartsman, "Boundary homogenization for trapping by patchy surfaces", J. Chem. Phys. 121, 11390 (2004).

[23] D. S. Grebenkov, M. Filoche, B. Sapoval, and M. Felici, "Diffusion-Reaction in Branched Structures: Theory and Application to the Lung Acinus", Phys. Rev. Lett. 94, 050602 (2005).

[24] D. S. Grebenkov, M. Filoche, and B. Sapoval, "Mathematical Basis for a General Theory of Laplacian Transport towards Irregular Interfaces", Phys. Rev. E 73, 021103 (2006).

[25] S. D. Traytak and W. Price, "Exact solution for anisotropic diffusion-controlled reactions with partially reflecting conditions", J. Chem. Phys. 127, 184508 (2007).

[26] P. C. Bressloff, B. A. Earnshaw, and M. J. Ward, "Diffusion of protein receptors on a cylindrical dendritic membrane with partially absorbing traps", SIAM J. Appl. Math. 68, 1223-1246 (2008).

[27] S. D. Lawley and J. P. Keener, "A New Derivation of Robin Boundary Conditions through Homogenization of a Stochastically Switching Boundary", SIAM J. Appl. Dyn. Sys. 14, 1845-1867 (2015).

[28] M. Galanti, D. Fanelli, S. D. Traytak, and F. Piazza, "Theory of diffusion-influenced reactions in complex geometries", Phys. Chem. Chem. Phys. 18, 15950-15954 (2016).

[29] A. E. Lindsay, A. J. Bernoff, and M. J. Ward, "First Passage Statistics for the Capture of a Brownian Particle by a Structured Spherical Target with Multiple Surface Traps", Multiscale Model. Simul. 15, 74-109 (2017).

[30] D. S. Grebenkov and G. Oshanin, "Diffusive escape through a narrow opening: new insights into a classic problem," Phys. Chem. Chem. Phys. 19, 2723-2739 (2017).

[31] A. Bernoff, A. Lindsay, and D. Schmidt, "Boundary Homogenization and Capture Time Distributions of Semipermeable Membranes with Periodic Patterns of Reactive Sites", Multiscale Model. Simul. 16, 1411-1447 (2018).

[32] D. S. Grebenkov and S. Traytak, "Semi-analytical computation of Laplacian Green functions in threedimensional domains with disconnected spherical boundaries," J. Comput. Phys. 379, 91-117 (2019).

[33] D. S. Grebenkov, "Imperfect Diffusion-Controlled Reactions", in Chemical Kinetics: Beyond the Textbook, Eds. K. Lindenberg, R. Metzler, and G. Oshanin (World Scientific, New Jersey, 2019).

[34] D. S. Grebenkov, "Paradigm shift in diffusion-mediated 
surface phenomena", Phys. Rev. Lett. 125, 078102 (2020).

[35] P. Lévy, Processus Stochastiques et Mouvement Brownien (Paris, Gauthier-Villard, 1965).

[36] K. Ito and H. P. McKean, Diffusion Processes and Their Sample Paths (Springer-Verlag, Berlin, 1965).

[37] M. Freidlin, Functional Integration and Partial Differential Equations (Annals of Mathematics Studies, Princeton University Press, Princeton, New Jersey, 1985).

[38] S. B. Yuste, E. Abad, and K. Lindenberg, "Exploration and trapping of mortal random walkers", Phys. Rev. Lett. 110, 220603 (2013).

[39] B. Meerson and S. Redner, "Mortality, redundancy, and diversity in stochastic search", Phys. Rev. Lett. 114, 198101 (2015).

[40] D. S. Grebenkov and J.-F. Rupprecht, "The escape problem for mortal walkers", J. Chem. Phys. 146, 084106 (2017).

[41] O. V. Bychuk and B. O'Shaugnessy, "Anomalous surface diffusion: A numerical study", J. Chem. Phys. 101, 772 (1994).

[42] O. V. Bychuk and B. O'Shaughnessy, "Anomalous Diffusion at Liquid Surfaces", Phys. Rev. Lett. 74, 1795 (1995).

[43] R. Walder, N. Nelson, and D. K. Schwartz, "Single Molecule Observations of Desorption-Mediated Diffusion at the Solid-Liquid Interface", Phys. Rev. Lett. 107, 156102 (2011).

[44] M. J. Skaug, J. Mabry, and D. K. Schwartz, "Intermittent Molecular Hopping at the Solid-Liquid Interface", Phys. Rev. Lett. 110, 256101 (2013).

[45] D. Wang, H. Wu, and D. K. Schwartz, "ThreeDimensional Tracking of Interfacial Hopping Diffusion", Phys. Rev. Lett. 119, 268001 (2017).

[46] A. V. Chechkin, I. M. Zaid, M. A. Lomholt, I. M. Sokolov, and R. Metzler, "Bulk-mediated surface diffusion along a cylinder: Propagators and crossovers", Phys. Rev. E 79, 040105(R) (2009).

[47] A. V. Chechkin, I. M. Zaid, M. A. Lomholt, I. M. Sokolov, and R. Metzler, "Effective surface motion on a reactive cylinder of particles that perform intermittent bulk diffusion", J. Chem. Phys. 134, 204116 (2011).

[48] A. V. Chechkin, I. M. Zaid, M. A. Lomholt, I. M. Sokolov, and R. Metzler, "Bulk-mediated diffusion on a planar surface: Full solution", Phys. Rev. E 86, 041101 (2012).

[49] A. M. Berezhkovskii, L. Dagdug, and S. M. Bezrukov, "A new approach to the problem of bulk-mediated surface diffusion", J. Chem. Phys. 143, 084103 (2015).

[50] A. M. Berezhkovskii, L. Dagdug, and S. M. Bezrukov, "Bulk-mediated surface transport in the presence of bias", J. Chem. Phys. 147, 014103 (2017).

[51] P. Mörters and Y. Peres, Brownian Motion (Cambridge Series in Statistical and Probabilistic Mathematics, Cambridge University Press, Cambridge, 2010).

[52] D. S. Grebenkov, "Residence times and other functionals of reflected Brownian motion", Phys. Rev. E 76, 041139 (2007).

[53] D. S. Grebenkov, "Probability distribution of the boundary local time of reflected Brownian motion in Euclidean domains", Phys. Rev. E 100, 062110 (2019).
[54] A. N. Borodin and P. Salminen, Handbook of Brownian Motion: Facts and Formulae (Birkhauser Verlag, BaselBoston-Berlin, 1996).

[55] L. Takacs, "On the local time of the Brownian motion", Ann. Appl. Probab. 5, 741 (1995).

[56] J. Randon-Furling and S. Redner, "Residence time near an absorbing set", J. Stat. Mech. 103205 (2018).

[57] W. Arendt, A. F. M. ter Elst, J. B. Kennedy, and M. Sauter, "The Dirichlet-to-Neumann operator via hidden compactness", J. Funct. Anal. 266, 1757-1786 (2014).

[58] D. Daners, "Non-positivity of the semigroup generated by the Dirichlet-to-Neumann operator", Positivity 18, 235-256 (2014).

[59] W. Arendt and A. F. M. ter Elst, "The Dirichlet-toNeumann Operator on Exterior Domains", Potential Anal. 43, 313-340 (2015).

[60] A. Hassell and V. Ivrii, "Spectral asymptotics for the semiclassical Dirichlet to Neumann operator", J. Spectr. Theory 7, 881-905 (2017).

[61] A. Girouard and I. Polterovich, "Spectral geometry of the Steklov problem", J. Spectr. Theory 7, 321-359 (2017).

[62] D. S. Grebenkov, Partially Reflected Brownian Motion: A Stochastic Approach to Transport Phenomena, in "Focus on Probability Theory", Ed. L. R. Velle, pp. 135-169 (Nova Science Publishers, 2006).

[63] D. S. Grebenkov, "Scaling Properties of the Spread Harmonic Measures", Fractals 14, 231-243 (2006).

[64] D. S. Grebenkov, "Analytical representations of the spread harmonic measure density", Phys. Rev. E 91, 052108 (2015).

[65] D. S. Grebenkov, "Diffusion toward non-overlapping partially reactive spherical traps: fresh insights onto classic problems," J. Chem. Phys. 152, 244108 (2020).

[66] V. Zaburdaev, S. Denisov, and J. Klafter, "Levy walks", Rev. Mod. Phys. 87, 483 (2015).

[67] D. S. Grebenkov, "Joint distribution of multiple boundary local times and related first-passage time problems with multiple targets" (accepted to J. Stat. Mech.)

[68] D. S. Grebenkov, "Spectral theory of imperfect diffusioncontrolled reactions on heterogeneous catalytic surfaces", J. Chem. Phys. 151, 104108 (2019).

[69] D. S. Grebenkov, "A physicist's guide to explicit summation formulas involving zeros of Bessel functions and related spectral sums" (submitted; available online arXiv:1904.11190v2)

[70] D. S. Grebenkov, R. Metzler, and G. Oshanin, "Strong defocusing of molecular reaction times results from an interplay of geometry and reaction control", Commun. Chem. 1, 96 (2018).

[71] D. C. Torney and H. M. McConnel, "Diffusion-limited reaction rate theory for two-dimensional systems", Proc. R. Soc. London A 387, 147-170 (1983).

[72] D. S. Grebenkov, "Reversible reactions controlled by surface diffusion on a sphere", J. Chem. Phys. 151, 154103 (2019).

[73] M. Smoluchowski, "Versuch einer Mathematischen Theorie der Koagulations Kinetic Kolloider Lösungen", Z. Phys. Chem. 92U, 129-168 (1917). 